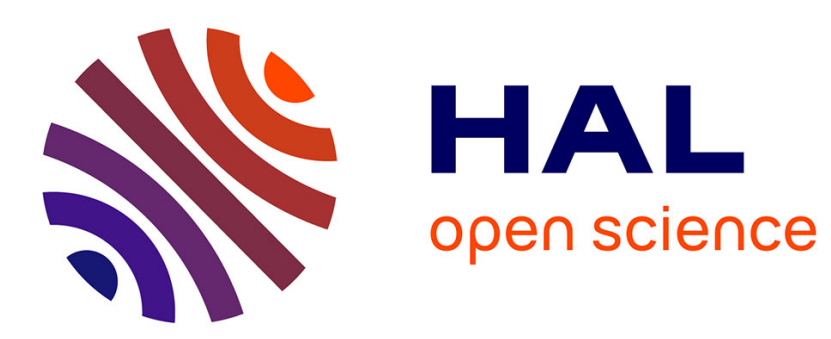

\title{
Accounting for Acoustic Damping in a Helmholtz Solver
}

Franchine Ni, Maxence Miguel-Brebion, Franck Nicoud, Thierry Poinsot

\section{To cite this version:}

Franchine Ni, Maxence Miguel-Brebion, Franck Nicoud, Thierry Poinsot. Accounting for Acoustic Damping in a Helmholtz Solver. AIAA Journal, 2017, 55 (4), pp.1205-1220. 10.2514/1.J055248 . hal-01553004

\section{HAL Id: hal-01553004 https://hal.science/hal-01553004}

Submitted on 3 Jul 2017

HAL is a multi-disciplinary open access archive for the deposit and dissemination of scientific research documents, whether they are published or not. The documents may come from teaching and research institutions in France or abroad, or from public or private research centers.
L'archive ouverte pluridisciplinaire HAL, est destinée au dépôt et à la diffusion de documents scientifiques de niveau recherche, publiés ou non, émanant des établissements d'enseignement et de recherche français ou étrangers, des laboratoires publics ou privés. 


\section{OATAO}

Open Archive Toulouse Archive Ouverte

\section{Open Archive TOULOUSE Archive Ouverte (OATAO)}

OATAO is an open access repository that collects the work of Toulouse researchers and makes it freely available over the web where possible.

This is an author-deposited version published in : http://oatao.univ-toulouse.fr/ Eprints ID : 17947

To link to this article : DOI:10.2514/1.J055248

URL : http://dx.doi.org/10.2514/1.J055248

To cite this version : Ni, Franchine and Miguel-Brebion, Maxence and Nicoud, Franck and Poinsot, Thierry Accounting for Acoustic Damping in a Helmholtz Solver. (2017) AIAA Journal, vol. $55\left(\mathrm{n}^{\circ} 4\right)$. pp. 1205-1220. ISSN 0001-1452

Any correspondence concerning this service should be sent to the repository administrator: staff-oatao@listes-diff.inp-toulouse.fr 


\title{
Accounting for Acoustic Damping in a Helmholtz Solver
}

\author{
F. Ni* \\ Centre Européen de Recherche et de Formation Avancée en Calcul Scientifique, \\ 31057 Toulouse, France \\ M. Miguel-Brebion \\ Institut de Mécanique des Fluides de Toulouse, 31400 Toulouse, France \\ F. Nicoud $\stackrel{\$}{ \pm}$ \\ Université de Montpellier, 34095 Montpellier, France \\ and \\ T. Poinsot胥 \\ Institut de Mécanique des Fluides de Toulouse, 31400 Toulouse, France
}

\begin{abstract}
DOI: $10.2514 / 1 . J 055248$
Thermoacoustic Helmholtz solvers provide a cheap and efficient way of predicting combustion instabilities. However, because they rely on the inviscid Euler equations at zero Mach number, they cannot properly describe the regions where aerodynamics may interact with acoustic waves, in the vicinity of dilution holes and injectors, for example. A methodology is presented to incorporate the effect of non-purely acoustic mechanisms into a threedimensional thermoacoustic Helmholtz solver. The zones where these mechanisms are important are modeled as two-port acoustic elements, and the corresponding matrices, which notably contain the dissipative effects due to acoustic-hydrodynamic interactions, are used as internal boundary conditions in the Helmholtz solver. The rest of the flow domain, where dissipation is negligible, is solved by the classical Helmholtz equation. With this method, the changes in eigenfrequency and eigenmode structure introduced by the acoustic-hydrodynamic effects are captured, while keeping the simplicity and efficiency of the Helmholtz solver. The methodology is successfully applied on an academic configuration, first with a simple diaphragm, then with an industrial swirler, with matrices measured from experiments and large-eddy simulation.
\end{abstract}

\section{Introduction}

$\mathbf{C}$ OMBUSTION instabilities are a major issue for modern combustion engines, such as rockets, turbojet engines, or gas turbines [1-3]. When the fluctuations of pressure and heat release are in phase, a coupling between flame and acoustics creates strong pressure oscillations in the combustor, potentially leading to an important structural damage or even a catastrophic engine failure. From an academic perspective, the phenomenon has been studied since the 1900s [4], but is still not fully understood. It is, therefore, an intense topic of research among the combustion community $[5,6]$.

For engine manufacturers, combustion instabilities must be avoided, preferably early enough in the design stage. There is consequently a need for numerical tools capable of predicting the stability of a combustor even before it is built. These tools range from simple network models that are fast and easy to implement [7-11] to more complex and time-consuming large-eddy-simulation ( $\overline{\mathrm{LES}}$ ) solvers [12-18]. In-between, linearized Navier-Stokes equation (LNSE) solvers and Helmholtz solvers provide a fair tradeoff between computational cost and fidelity. They are suitable for complex three-dimensional (3-D) industrial geometries, but are simpler and faster than LES. LNSEs have the merit of directly including acoustic-hydrodynamic effects as demonstrated for simple two-dimensional (2-D) and 3-D configurations [19-21], but to the authors' knowledge, most LNSE studies focus on the propagation of acoustic fluctuations in nonreactive flows. In the context of combustion instabilities, we are more interested in determining the eigenmodes of a reactive flow, and this is well achieved by a Helmholtz solver. Compared to LNSE solvers, Helmholtz codes solve the linearized inviscid Euler equations with a baseline flow at rest, and require less computational power and less refined meshes. This simplicity comes at a price, because any mechanism implying interactions with fluid viscosity, heat transfer, or hydrodynamics is left apart. In a typical combustor (Fig. 1), even if the zero Mach-number assumption is justified in most of the domain, these mechanisms could still have an acoustic impact (Table 1) [22-36].

Some of these contributions are well known and can be accounted for in the Helmholtz solution. This is the case of the acoustic losses created at thermoviscous boundary layers. When acoustic waves interact with the boundary layers, acoustic energy is dissipated through shear stress and heat losses. A synthetic model has been proposed by Searby et al. [25] and is of great interest because it can be used to postprocess a Helmholtz computation. The treatment extracts the damping rate due to thermoviscous effects for any standing mode computed by the dissipation-free Helmholtz solver.

One interesting observation in the study of Searby et al. [25] is that thermoviscous damping scales as the square root of the frequency, and is therefore a high-frequency phenomenon, more common in rocket engines than in turbojet engines. Most of the unstable modes encountered in turbine combustion chambers have low-enough frequencies to neglect thermoviscous damping. At these frequencies, another mechanism is at stake. When a mean flow is present, a shear layer is created at sudden section changes and can extract energy from the acoustics by converting it into vorticity $[28,31,37,38]$. In the case of perforated plates, such as the ones involved in cooling the combustor walls (see Fig. 1), the impact on acoustics can be quantified using, for example, Howe's model [28], as recalled in Appendix A.

Howe's model and its counterparts [29,30,39] can be used to represent perforated plates as homogeneous boundary conditions in a Helmholtz solver [32] or in a computational fluid dynamics (CFD) code [30]. For more complex systems, such as swirlers, no analytical model is available to describe the acoustic-hydrodynamic effects. 


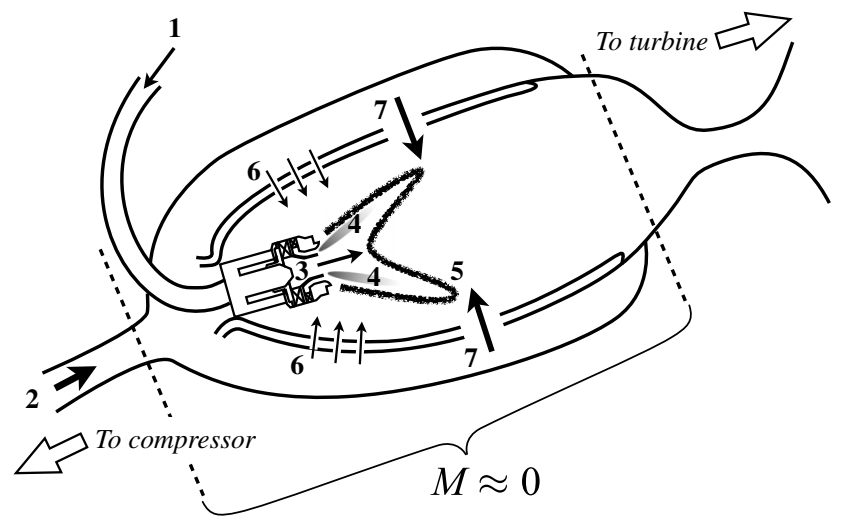

1 Fuel supply

2 Air supply

3 Swirler

4 Liquid fuel spray

5 Flame

6 Multiperforated plate

7 Dilution hole

Fig. 1 Typical turbojet combustor design and its components.

Still, provided that such a complex system behaves like a linear timeinvariant causal system, it can be abstracted as a $2 \times 2$ matrix in the frequency domain. The latter can be measured experimentally $[40,41]$ or numerically [42]. The method is well known, and has often been used in the acoustic analysis of ducts and mufflers [35,43-45], and flame dynamics [46-48]. Some studies also report results on swirlers [34] and heat exchangers [49]. The equivalent matrix can be plugged into an acoustic network to provide results for complex configurations by decomposing them into simpler elements $[\underline{43}, \underline{50,51]}$.

In this paper, we propose to incorporate this matrix formalism into a Helmholtz solver, in a similar way to what was proposed by Campa and Camporeale [52] and Laera et al. [53]. With this approach, the acoustic impact of non-purely acoustic mechanisms can be reproduced in the Helmholtz solver for very general systems (for example, the swirler and the dilution holes in Fig. 1), so that the result is more representative of an actual combustor. Whereas the work of Laera et al. [53] uses the matrix formalism mainly to describe flame-acoustics coupling, the present work deals with the dissipative effects due to acoustichydrodynamic interaction. The methodology is applied to an academic configuration containing a dissipative element (first a diaphragm, then a swirler), whose dissipative behavior is quantified experimentally and by the proposed enhanced Helmholtz-solver approach. The target configuration is also computed with LES so that comparisons between the Helmholtz solver, the LES computation, and experimental results can be provided.

The paper is structured as follows. The methodology and its practical implementation in a Helmholtz solver are presented in Sec. II. To check its validity, comparisons between LES, experiments, and Helmholtz-solver results are performed for an academic configuration. The overall validation strategy and the target configuration are described in Sec. III. The results are then presented for a diaphragm in Sec. IV, and for an industrial swirler in Sec. V.

\section{Description of the Methodology}

As briefly stated in the Introduction, we propose to model the dissipative behavior of an element inside an inviscid domain by

Table 1 Examples of non-purely acoustic mechanisms present in a typical combustor (Fig. 1)

\begin{tabular}{|c|c|c|}
\hline Mechanism & Location in combustor & $\begin{array}{c}\text { Available } \\
\text { models }\end{array}$ \\
\hline $\begin{array}{l}\text { Acoustic losses in thermal and } \\
\text { viscous boundary layers }\end{array}$ & $\begin{array}{l}\text { No-slip walls, } \\
\text { nonadiabatic walls }\end{array}$ & [22-25] \\
\hline $\begin{array}{l}\text { Drag/thermal losses due to liquid } \\
\text { particles }\end{array}$ & Liquid spray & {$[\underline{26}, \underline{27}]$} \\
\hline $\begin{array}{l}\text { Acoustic-hydrodynamic interactions } \\
\text { at perforated plate }\end{array}$ & Multiperforated plates & {$[\underline{28}-\underline{33}]$} \\
\hline $\begin{array}{l}\text { Acoustic-hydrodynamic interactions } \\
\text { at other elements }\end{array}$ & $\begin{array}{l}\text { Swirler, dilution holes, } \\
\text { T-junctions }\end{array}$ & {$[\underline{21}, \underline{34}-\underline{36}]$} \\
\hline
\end{tabular}

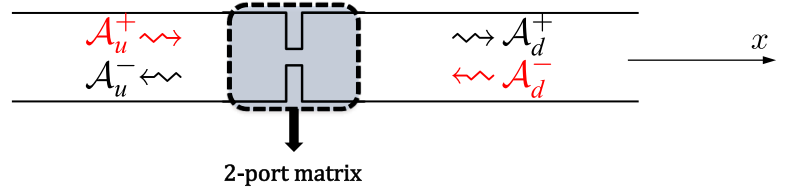

Fig. 2 Modelization of an acoustic system (here, a slit) as a two-port filter.

measuring its two-port matrix. The concept is well defined when plane modes are considered. The acoustic states up- and downstream of the dissipative zone are fully described by two quantities, for example, $p^{\prime}$ and $\rho_{o} c_{o} u^{\prime}$, the acoustic pressure and velocity fluctuations, or $\mathcal{A}^{+}=$ $\left(p^{\prime}+\rho_{o} * c_{o} * u^{\prime}\right) / 2$ and $\mathcal{A}^{-}=\left(p^{\prime}-\rho_{o} * c_{o} * u^{\prime}\right) / 2$ the right and left traveling waves. The direction of the longitudinal axis $x$ is chosen so that $u^{\prime}>0$ corresponds to a velocity perturbation moving toward the right. For a linear system, the upstream and downstream states are connected by a $2 \times 2$ matrix; see Fig. 2 . When $\mathcal{A}^{+}$and $\mathcal{A}^{-}$are used instead, this matrix is called the scattering matrix. Other conventions exist, and some are listed in Table 2 .

In the following, we show how the damping effect of acoustichydrodynamic systems can be included in a typical 3-D thermoacoustics Helmholtz solver (the AVSP solver [54] in this study). For this purpose, the zones that cannot be described by acoustics only are removed from the domain where the Helmholtz equation is solved, and replaced by their $2 \times 2$ matrix (Fig. 3). This operation requires the coupling of a $3-D$ Helmholtz solver with a twoport matrix, which is achieved by a matrix boundary condition (MBC) described next. Dissipative effects located at the boundaries of the total fluid domain, such as radiation, are modeled with classical impedances (Fig. 3).

The acoustic quantities are decomposed as $p^{\prime}(\boldsymbol{x}, t)=\Re\left[\hat{p}(\boldsymbol{x}) e^{-i \omega t}\right]$ and $\boldsymbol{u}^{\prime}(\boldsymbol{x}, t)=\mathfrak{R}\left[\hat{\boldsymbol{u}}(\boldsymbol{x}) e^{-i \omega t}\right]$, assuming a harmonic time dependence and a spatially varying amplitude. Their behavior in the frequency domain is determined by the Helmholtz equation (1) (given here in the absence of a source term) and the linearized momentum equation (2).

Table 2 Examples of acoustic two-port formulation (from [34])

\begin{tabular}{lcc}
\hline \hline Name & State variables & Defining equation \\
\hline Transfer matrix & $p^{\prime}, u^{\prime}$ & {$\left[\begin{array}{c}p_{d}^{\prime} /\left(\rho_{o, d} c_{o, d}\right) \\
u_{d}^{\prime}\end{array}\right] \equiv T_{a}\left[\begin{array}{c}p_{u}^{\prime} /\left(\rho_{o, u} c_{o, u}\right) \\
u_{u}^{\prime}\end{array}\right]$} \\
Scattering matrix & $\mathcal{A}^{+}, \mathcal{A}^{-}$ & $\left(\begin{array}{c}\mathcal{A}_{d}^{+} \\
\mathcal{A}_{u}^{-}\end{array}\right) \equiv S\left(\begin{array}{c}\mathcal{A}_{u}^{+} \\
\mathcal{A}_{d}^{-}\end{array}\right)$ \\
Mobility matrix & $p^{\prime}, u^{\prime}$ & $\left(\begin{array}{c}u_{u}^{\prime} \\
u_{d}^{\prime}\end{array}\right) \equiv M\left[\begin{array}{l}p_{u}^{\prime} /\left(\rho_{o, u} c_{o, u}\right) \\
p_{d}^{\prime} /\left(\rho_{o, d} c_{o, d}\right)\end{array}\right]$ \\
\hline \hline
\end{tabular}




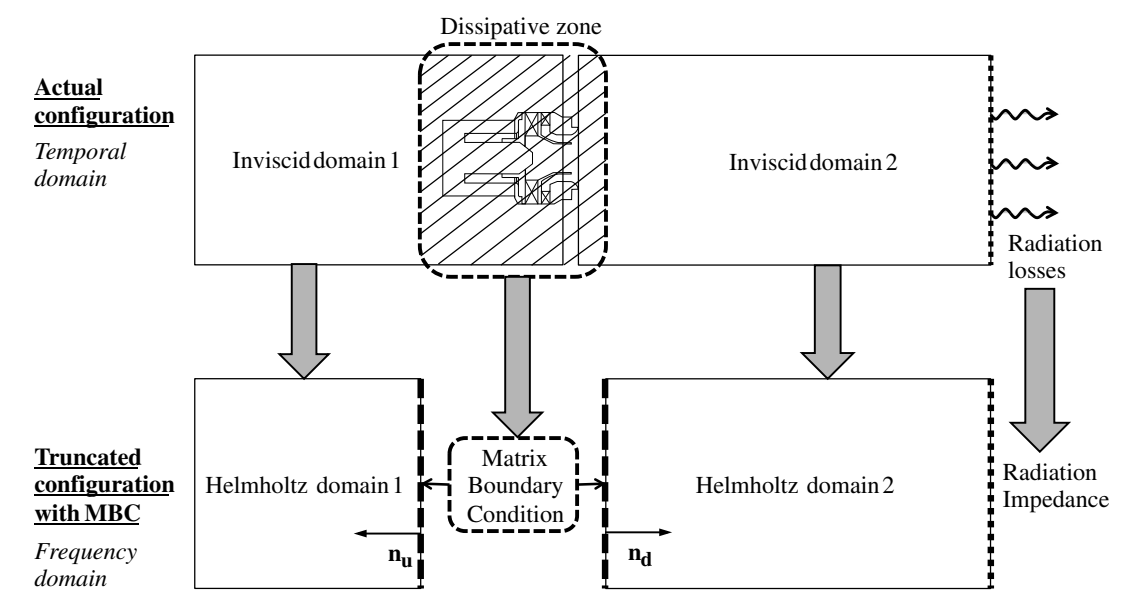

Fig. 3 Locally dissipative elements inside a globally inviscid domain are modeled by two-port matrices.

$$
\begin{gathered}
\gamma P_{o}(\boldsymbol{x}) \nabla \cdot\left[\frac{1}{\rho_{o}(\boldsymbol{x})} \nabla \hat{p}(\boldsymbol{x})\right]+\omega^{2} \hat{p}(\boldsymbol{x})=0 \\
\hat{\boldsymbol{u}}=\frac{1}{\rho_{o} i \omega} \nabla \hat{p}
\end{gathered}
$$

with $\gamma$ the ratio of specific heats (constant, equal to 1.4 for ambient air) and $P_{o}$ the temporal average of the pressure. The Helmholtz eigenvalue problem made up of Eqs. (1) and (2) is discretized at nodes and solved by the AVSP solver [54] with a finite volume strategy. To close the problem at boundaries, information about the pressure gradient along the boundary normal must be provided in the form of pressure boundary conditions (Fig. 4). To account for the two-port matrix in the truncated flow domain, a new boundary condition should therefore be defined, which relates the pressure fluctuation $\hat{p}$ to its gradient. This is obtained by combining the mobility-matrix formulation (last row of Table 2) with the linearized momentum equation (2).

$$
\left\{\begin{array}{l}
\nabla \hat{p}_{u} \cdot \boldsymbol{n}_{u}=M_{11} \frac{i \omega}{c_{o, u}} \hat{p}_{u}+M_{12} \frac{i \omega}{c_{o, d}} \frac{\rho_{o, u}}{\rho_{o, d}} \hat{p}_{d} \\
\nabla \hat{p}_{d} \cdot \boldsymbol{n}_{d}=M_{22} \frac{i \omega}{c_{o, d}} \hat{p}_{d}+M_{21} \frac{i \omega}{c_{o, u}} \frac{\rho_{o, d}}{\rho_{o, u}} \hat{p}_{u}
\end{array}\right.
$$

in which the upstream and downstream boundary normal vectors $\boldsymbol{n}_{u}$ and $\boldsymbol{n}_{d}$ are defined with an inward normal convention (Fig. 3). System (3) defines a pair of Robin conditions suited to represent the effect of a non-purely acoustic element, including its dissipation (Fig. 3). These boundary conditions are applied pointwise to a pair of patches. To link the noncoincident upstream and downstream meshes, the nodes of each patch are projected onto the other, and the field of $\hat{p}$ is linearly interpolated on the projected nodes.

With the MBC, it is possible to account for nonacoustic elements in a Helmholtz solver with the following strategy:

1) Remove all dissipative/nonacoustic zones from the domain. Note that this is different from the LNSE approach, in which the dissipative elements need to be meshed. Once the dissipation matrices are obtained, the MBC procedure runs with lighter and simpler meshes than the LNSE ones.

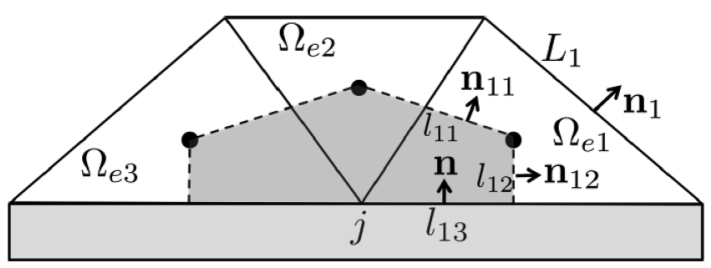

Fig. 4 Discretization of a 2-D boundary node in AVSP (dual cell in grey).
2) Link the remaining inviscid domains with a pair of internal MBCs, representative of the element previously removed.

3) Solve the discretized Helmholtz eigenproblem with the MBC internal boundaries. Note that the introduction of MBC makes the Helmholtz eigenproblem nonlinear with respect to the eigenvalues, because the eigenproblem operator at boundaries now depends on the eigenfrequency; see Eq. (3). The problem remains, however, linear with respect to the eigenvectors (pressure mode shape), because no dependency with the amplitude is considered in this study. The nonlinear eigenvalue search is treated in AVSP with a fixed-point loop described in [54].

As a verification test, it was checked (not shown) that the MBC implementation matches the analytical eigenmodes and eigenfrequencies in cases in which analytical solutions are available [e.g., 2-D tubes connected by a simple identity matrix or the matrix corresponding to Howe's model, Eq. (A1)].

\section{Validation Strategy}

In the configuration described in Fig. 3 , the conventional Helmholtz solver cannot account for the dissipative effects created by acoustic-hydrodynamic interactions at the swirler even if the whole geometry of the latter is represented by the finite volume mesh. However, adding the MBC should improve the results. To apply the MBC methodology, the matrix of the dissipative element is required and must be retrieved either experimentally or numerically. To check whether the resulting eigenfrequencies and eigenmodes are "better" than without MBC, they are compared to reference eigenmodes and eigenfrequencies, which are again either computed by LES or measured in experiments.

This section explains how the MBC methodology is applied to a target configuration described in Sec. III.A. For this configuration, the matrix data and reference eigenpairs are obtained from experiments (Sec. III.B) and from LES (Sec. III.C). The matrices are used to define $\mathrm{MBC}$ in the Helmholtz computation of the truncated domain. The corresponding numerical setup is presented in Sec. III.D.

To check the validity of the MBC methodology, four types of comparisons, called C1, C2-LES, C2-EXP, and C3 in Fig. $\underline{5}$, are performed:

1) C1: Matrices obtained from the experiments and from the LES are compared, to check their validity and robustness to either numerical or experimental artifacts.

2) C2-LES: The LES-matrix data are used as an input of the Helmholtz solver with MBC. The resulting eigenfrequencies and eigenmodes are compared with the LES values, and with the results of the Helmholtz solver without MBC.

3) C2-EXP: The experimental-matrix data are used as an input of the Helmholtz solver with MBC. The resulting eigenfrequencies and eigenmodes are compared with the experimental values and with the results of the Helmholtz solver without MBC. 


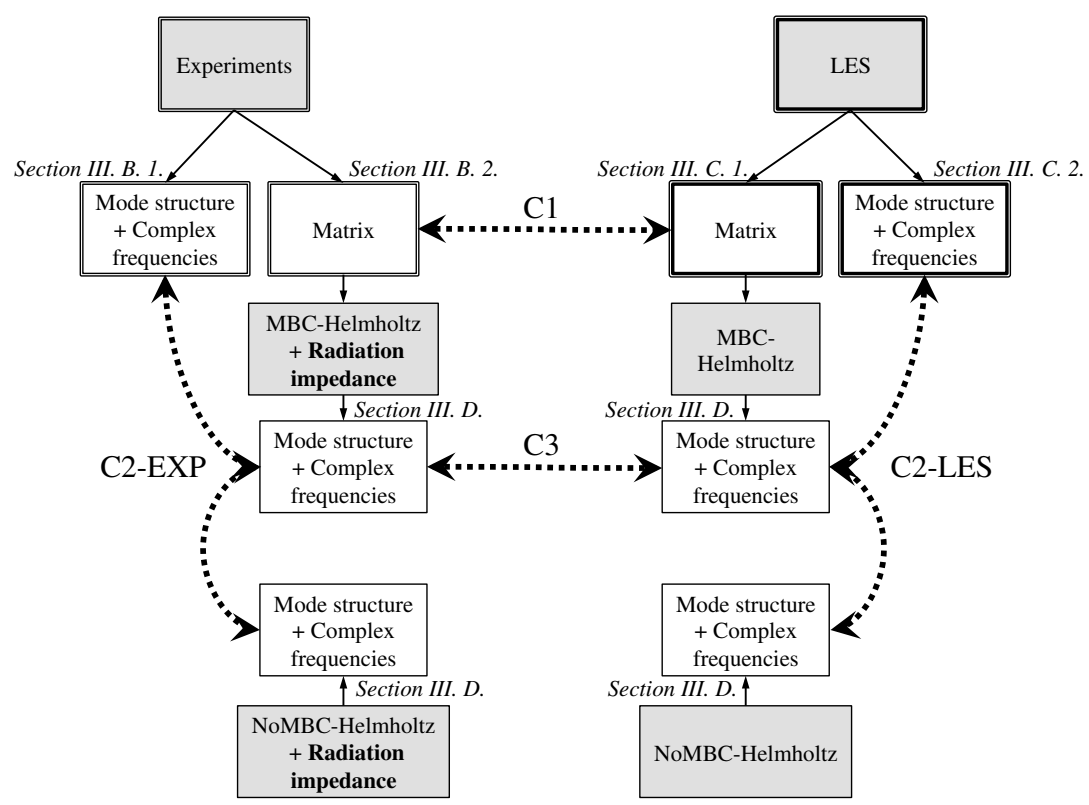

Fig. 5 MBC validation strategy with its four axes of comparison.

4) C3: The MBC Helmholtz-solver results from the LES matrix and from the experimental matrix are compared to check the sensitivity of the results to the input data used to model the nonacoustic element.

\section{A. Target Setup}

Two dissipative elements are considered in this study: a diaphragm (Fig. 6a) and a swirler (Fig. 6b). They are installed in an experimental rig composed of cylindrical ducts of different lengths (Fig. 7). The experiments are run at ambient temperature $T_{o}=299 \overline{\mathrm{K}}$ and ambient pressure $P_{o}=101,550 \mathrm{~Pa}$ with air (specific ratio of $\gamma=$ 1.4 and molecular mass of $W=28.97 \mathrm{~g} / \mathrm{mol}$ ).

Airflow is driven by eight uniformly distributed radial injectors. To perform acoustic measurements, the rig airflow is excited by a loudspeaker (Focal ISN 100) fixed in a PVC airtight module. This loudspeaker is placed at the end of the inlet tube for the acquisition of two-port matrices (Fig. 7a), or on the side of the tube for the acquisition of acoustic modes (Fig. 7b). Six microphones (Brïel \& Kjær 1/4 in. Type 4954-B) measure the acoustic pressure in the rig: three upstream and three downstream of the dissipative element.

Three PVC tubes (T1, T2, T3) with the same cross section but different lengths are used in the experimental studies. Their inner radius is $0.041 \mathrm{~m}$, and lengths are $l_{1}=0.26 \mathrm{~m}, l_{2}=0.34 \mathrm{~m}$, and $l_{3}=0.56 \mathrm{~m}$, respectively. End corrections of $\delta_{\text {in }}=0.010 \mathrm{~m}$ and $\delta_{\text {out }}=0.025 \mathrm{~m}$ were used to match the experimental and Helmholtzsolver eigenfrequencies, in the absence of mean flow.

$\mathrm{T} 1, \mathrm{~T} 2$, and $\mathrm{T} 3$ are combined to obtain four independent configurations for the experimental measurement of the matrix, as explained in Sec. III.B.2. Regarding the measurement of acoustic modes and frequencies, only tubes $\mathrm{T} 3$ and $\mathrm{T} 2$ are used, and are placed upstream and downstream of the element of interest, respectively

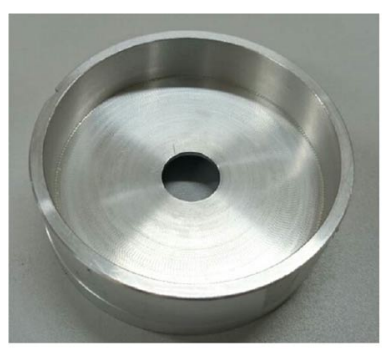

a) Diaphragm

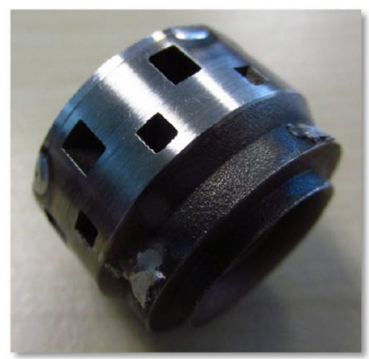

b) Swirler
Fig. 6 Acoustic elements: a) diaphragm and b) swirler.
(Fig. 7b). In this case, the loudspeaker is also moved to the side of the upstream duct, so as not to alter the closed boundary condition at the upstream end.

Acoustic matrices and modes are determined for three different flow rates characterized by their bulk velocities: $U=0.0,0.17$, and $0.34 \mathrm{~m} / \mathrm{s}$. These bulk velocities, imposed by a mass-flow meter, are controlled with a precision of $2 \%$ by using hot-wire-based velocityprofile measurements. In every case, the Mach number is lower than 0.001, and Mach-number effects in the inviscid zones can be neglected.

The acquisition system is based on the LabVIEW software and its associated multichannel acquisition board. Measurements are performed with harmonic excitations, for frequencies ranging from 50 up to $1000 \mathrm{~Hz}$, well below the cutoff frequency of $2500 \mathrm{~Hz}$. This ensures that only plane waves exist in the ducts. For each frequency and configuration, samples of $2 \mathrm{~s}$ are recorded at a sampling rate of $10 \mathrm{kHz}$.

\section{B. Experimental Matrices and Modes}

To perform comparison C2-EXP (Fig. 5), the acoustic modes and acoustic matrices are measured experimentally.

\section{Frequencies and Dissipation of Acoustic Modes in the Experiment}

The first step is to measure the frequencies and damping rates of the acoustic modes in the experiment (Fig. 7b). To do this, the test rig is excited by a loudspeaker producing a monochromatic harmonic signal at a constant voltage, and the resulting pressure waves are measured by microphones for a discrete set of frequencies. Under the plane-wave assumption, the complex acoustic-pressure amplitude at location $z_{j}$ can be expressed as

$$
\begin{gathered}
\hat{p}\left(z_{j}\right)=a_{j} e^{i \phi_{j}}=\mathcal{A}^{+} e^{i k^{+} z_{j}}+\mathcal{A}^{-} e^{-i k^{-} z_{j}} \\
\rho_{o} c_{o} \hat{u}\left(z_{j}\right)=\mathcal{A}^{+} e^{i k^{+} z_{j}}-\mathcal{A}^{-} e^{-i k^{-} z_{j}}
\end{gathered}
$$

in which $\mathcal{A}^{+}$and $\mathcal{A}^{-}$are the amplitudes of the right and left traveling waves, respectively. The origin $z=0$ of the axial coordinate $z_{j}$ in the duct is chosen at the center of the acoustic element (diaphragm or swirler).

By measuring the complex acoustic pressure at different locations, the wave amplitudes are retrieved at each frequency, thanks to the multimicrophone technique $[\underline{41}, \underline{55}]$. 
Section where the dissipative element is installed

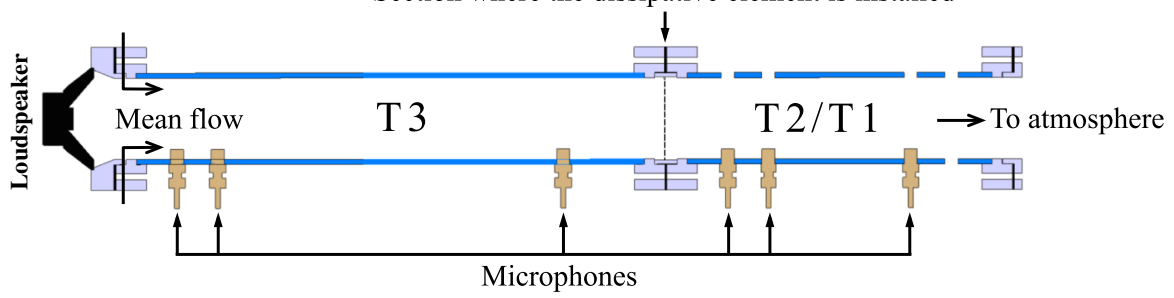

a) Matrix measurement. The downstream part can be modified as shown in Fig. 9

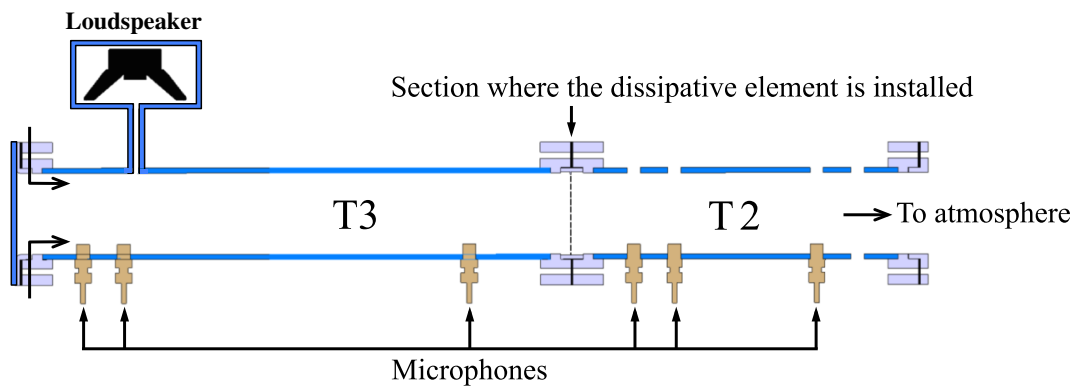

b) Acoustic mode measurement

Fig. 7 Sketch of the experimental setup for a) matrices and b) acoustic modes measurement.

$$
\begin{gathered}
\underbrace{\left(\begin{array}{cc}
e^{i k^{+} z_{1}} & e^{-i k^{-} z_{1}} \\
\ldots & \ldots \\
e^{i k^{+} z_{J}} & e^{-i k^{-} z_{J}}
\end{array}\right)}_{D} \underbrace{\left(\begin{array}{c}
\mathcal{A}^{+} \\
\mathcal{A}^{-}
\end{array}\right)}_{x}=\underbrace{\left(\begin{array}{c}
a_{1} e^{i \phi_{1}} \\
\ldots \\
a_{J} e^{i \phi_{J}}
\end{array}\right)}_{b} \\
\tilde{x}=\left(D^{\dagger} D\right)^{-1} D^{\dagger} b
\end{gathered}
$$

System (6) is overdetermined when more than two microphones are used, and is inverted with a least-squares approach as done in Eq. (7).

The wave amplitudes are combined to reconstruct $\mathcal{E}$, the periodaveraged acoustic energy integrated over the volume $V$ of the rig, as defined in Eq. (ㅇ).

$$
\mathcal{E}=\int_{V}\left(\frac{1}{4 \rho_{o} c_{o}^{2}}|\hat{p}(z)|^{2}+\frac{1}{4} \rho_{o}|\hat{u}(z)|^{2}\right) \mathrm{d} V
$$

For plane waves at low Mach number in a forced harmonic regime, the wave amplitudes are constant in each inviscid rig portion, and the term in the integral of Eq. (8) is independent of the coordinate $z$. The integration can thus be performed separately over the upstream and downstream volumes $V_{u}$ and $V_{d}$ to obtain Eq. (9).

$$
\begin{gathered}
\mathcal{E}(\omega)=V_{u}\left\{\frac{1}{2 \rho_{o} c_{o}^{2}}\left[\left|\mathcal{A}_{u}^{+}(\omega)\right|^{2}+\left|\mathcal{A}_{u}^{-}(\omega)\right|^{2}\right]\right\} \\
+V_{d}\left\{\frac{1}{2 \rho_{o} c_{o}^{2}}\left[\left|\mathcal{A}_{d}^{+}(\omega)\right|^{2}+\left|\mathcal{A}_{d}^{-}(\omega)\right|^{2}\right]\right\}
\end{gathered}
$$

The spectrum of acoustic energy $\mathcal{E}(\omega)$ contains information about the eigenfrequencies of the system, in the form of resonance peaks (Fig. 8). A fit is performed on these peaks to retrieve the complex eigenfrequencies $[56,57]$ based on the idea that the complex wave amplitudes $\mathcal{A}^{+}$and $\overline{\mathcal{A}}^{-}$in the system are solutions of a damped oscillator equation of the form:

$$
\ddot{\eta}(t)-2 \omega_{0 i} \dot{\eta}(t)+\omega_{0 r}^{2} \eta(t)=F
$$

in which $F=\hat{F} e^{-i \omega t}$ is the harmonic-forcing term, and $\eta(t)$ is to be replaced by $\mathcal{A}^{+} e^{-i \omega t}$ or $\mathcal{A}^{-} e^{-i \omega t}$. The forcing angular frequency $\omega$ is to be distinguished from the unknown complex angular eigenfrequency $\omega_{0}=2 \pi f_{0}=\omega_{0 r}+i \omega_{0 i}$, whose imaginary part $\omega_{0 i}$ is the growth rate. Equation (10) is valid only for negative values of $\omega_{0 i}$, corresponding to purely damped acoustics, as is the case here.

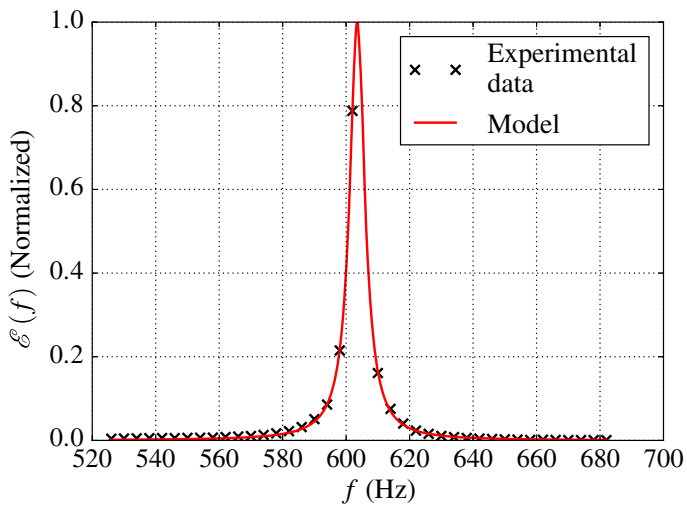

a) Mode 4 at $(603.5 \pm 0.1)-\mathrm{i}(2.9 \pm 0.1) \mathrm{Hz}$

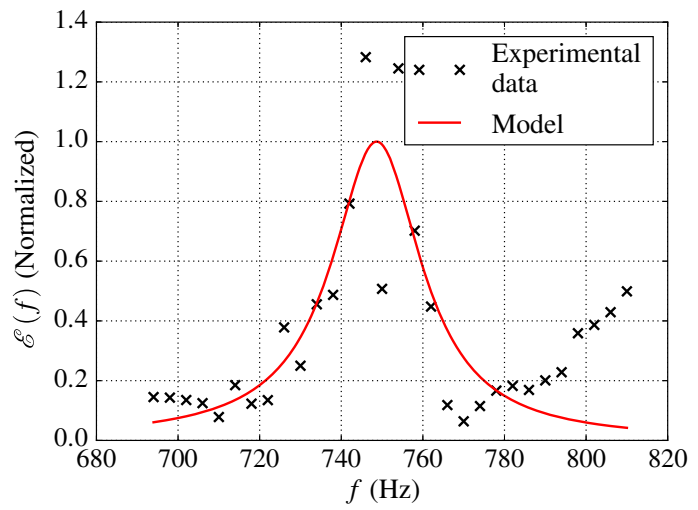

b) Mode 5 at $(748.9 \pm 3.5)-\mathrm{i}(13.4 \pm 5.0) \mathrm{Hz}$

Fig. $8 \mathcal{E}(\omega)$ spectra for two modes of the diaphragm case at $U=0.17 \mathrm{~m} / \mathrm{s}$. 
Transposing Eq. (10) in the Fourier domain gives an expression for $\mathcal{A}^{+}$and $\mathcal{A}^{-}$as a function of $\omega_{0 r}$ and $\omega_{0 i}$, which is combined with Eq. (9) to yield an expression for $\mathcal{E}(\omega)$ :

$$
\mathcal{E}_{\text {theo }}(\omega)=\frac{1}{2 \pi} \frac{G}{\left(\omega_{0 r}^{2}-\omega^{2}\right)^{2}+4 \omega_{0 i}^{2} \omega^{2}}
$$

with $G=|\hat{F}|^{2}\left(V_{\text {up }}+V_{\text {down }}\right) /\left(2 \rho_{o} c_{o}^{2}\right)$. This is the form used to fit $\mathcal{E}(\omega)$. The three parameters $G, \omega_{0 r}$, and $\omega_{0 i}$ are tuned so that Eq. (11) produces the best possible match of the measured spectrum $\mathcal{E}(\bar{\omega})$. This method is equivalent to but more precise than measuring the width at half-height of the peaks $[57,58]$. The quality of the fit is assessed by computing the uncertainty on $f_{0}=\left(\omega_{0 r}+i \omega_{0 i}\right) /(2 \pi)$ with a $95 \%$ confidence interval. This uncertainty displayed as a \pm value in the captions of Fig. $\underline{8}$ is low when the data are well fitted (Fig. 8a), and increases as soon as the quality of the fit deteriorates, when the signal is noisy, for example (Fig. $\underline{8 b}$ ). In Fig. $\underline{8}, \mathcal{E}(\omega)$ is normalized by the value fitted at $\omega_{0}$.

Once the eigenfrequencies are estimated, the associated eigenmodes and acoustic fields can easily be reconstructed using Eqs. (ㅁ) and (ㅁ) .

\section{Experimental Two-Port Matrices}

The two-port matrix is reconstructed following [59], with the scattering-matrix formalism (see Table 2). This requires at least two linearly independent configurations. More robust results are obtained by using four independent configurations. In this study, this is achieved by combining in different ways the three ducts T1, $\mathrm{T} 2$, and T3, and switching the outlet impedance from open to closed (Fig. 9). For each configuration, the wave amplitudes are measured upstream and downstream of the acoustic element of interest with the multimicrophone technique exposed previously. As in the multimicrophone technique, an overdetermined system (12) is defined from the four independent configurations of Fig. 9 with the matrix coefficients $S=\left(\begin{array}{cc}t_{u} & r_{d} \\ r_{u} & t_{d}\end{array}\right)$ as unknowns, and inverted with a least-squares approach.

$$
\begin{gathered}
\underbrace{\left(\begin{array}{cccc}
\mathcal{A}_{u, 1}^{+} & \mathcal{A}_{d, 1}^{-} & 0 & 0 \\
0 & 0 & \mathcal{A}_{u, 1}^{+} & \mathcal{A}_{d, 1}^{-} \\
\vdots & \vdots & \vdots & \vdots \\
\mathcal{A}_{u, j}^{+} & \mathcal{A}_{d, j}^{-} & 0 & 0 \\
0 & 0 & \mathcal{A}_{u, j}^{+} & \mathcal{A}_{d, j}^{-} \\
\vdots & \vdots & \vdots & \vdots \\
\mathcal{A}_{u, N}^{+} & \mathcal{A}_{d, N}^{-} & 0 & 0 \\
0 & 0 & \mathcal{A}_{u, N}^{+} & \mathcal{A}_{d, N}^{-}
\end{array}\right)} \underbrace{\left(\begin{array}{c}
t_{u} \\
r_{d} \\
r_{u} \\
t_{d}
\end{array}\right)}_{s}=\underbrace{\left(\begin{array}{c}
\mathcal{A}_{d, 1}^{+} \\
\mathcal{A}_{u, 1}^{-} \\
\vdots \\
\mathcal{A}_{d, j}^{+} \\
\mathcal{A}_{u, j}^{-} \\
\vdots \\
\mathcal{A}_{d, N}^{+} \\
\mathcal{A}_{u, N}^{-}
\end{array}\right)}_{a} \\
\tilde{s}=\left(H^{\dagger} H\right)^{-1} H^{\dagger} a, \quad \operatorname{err}_{\text {relative }}=\|H \tilde{s}-a\| /\|a\|
\end{gathered}
$$

Equation (13) provides the scattering-matrix coefficients, as well as an estimation of the least-squares error. The second index $j$ runs from 1 to $N=4$, and denotes the $i$ th independent state. This

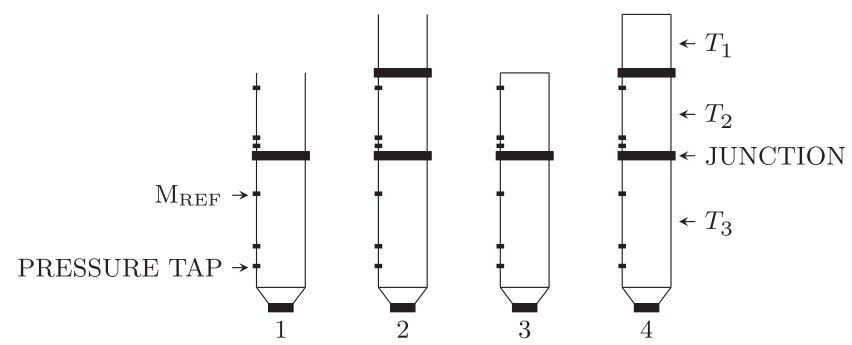

Fig. 9 Configurations used to measure the matrices in the experiments. scattering matrix is easily converted into the mobility matrix required for the MBC.

\section{LES Matrices and Modes}

The two-port matrices and acoustic eigenmodes can also be determined through LES computations performed with the AVBP solver, codeveloped by the Centre Européen de Recherche et de Formation Avancée en Calcul Scientifique (CERFACS) and the Institut Français du Pétrole Energies Nouvelles (IFPEN) [60]. This solver is widely used and has been validated in numerous situations $[15,18,30,61-64]$, to cite a few. The results will serve to check the experiments in comparison $\mathrm{C} 1$ and to perform comparison C2-LES (Fig. 5). The numerical parameters and boundary conditions based on the models in [65-68] are given in Appendix B. This section presents the methodology used to obtain the numerical matrices and eigenmodes.

\section{LES Matrices}

To compute the two-port matrix from the LES data, two independent harmonic-forcing states are used $[59,69]$. In state 1 , the acoustic element is excited from the inlet, with a nonreflective condition prescribed at the outlet. In state 2 , the acoustic element is excited from the outlet, with a nonreflective inlet. This is the only difference with the approach used in the experiments, in which the independent states were obtained by changing the outlet impedance, while always forcing at the inlet.

The geometry and overview of the mesh are shown in Fig. 10 for the diaphragm, and Fig. 11 for the swirler. The duct lengths used in the LES do not have to correspond to the experimental tubes. They were chosen long enough to let acoustic waves become onedimensional away from the dissipative elements, and short enough to minimize computational times. The swirler vanes are discretized with 18 points along the smallest dimension, and the diaphragm is meshed with 40 points in the diameter. Both meshes contain a few million nodes ( 2.5 million points for the swirler and 1 million points for the diaphragm).

The pressure is measured at probes equidistributed along the pipe circumference for several axial locations. For the diaphragm, 7 upstream stations and 11 downstream stations are used. For the swirler, only four upstream stations and five downstream stations are used because the hydrodynamic fluctuations created by the swirler extend further than in the diaphragm case. The wave amplitudes and the scattering matrix are then reconstructed with the same method as for the experimental data.

\section{LES Eigenmodes and Damping Rates}

The experimental data provide references for the real eigenfrequencies and the associated mode structure. This was obtained by forcing the experiment at hundreds of frequencies and

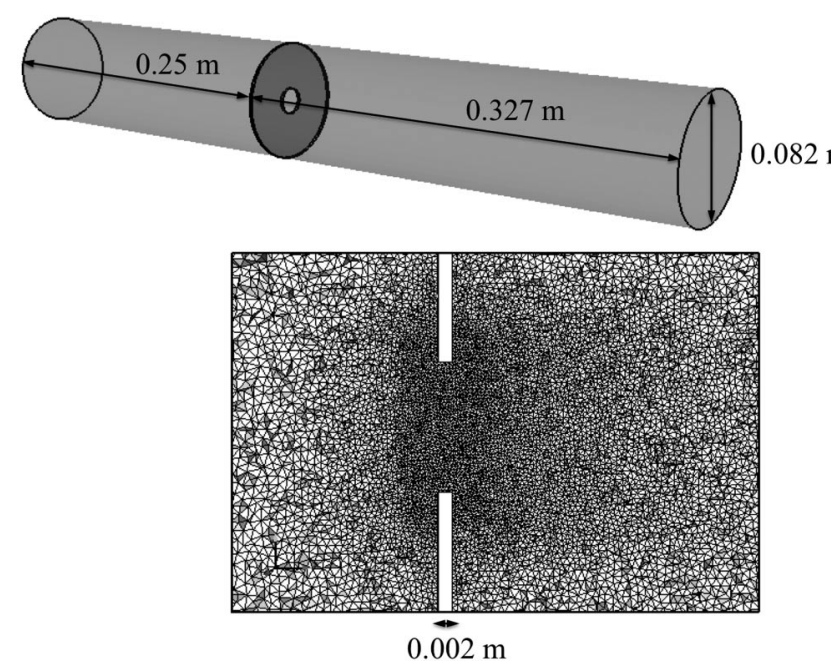

Fig. 10 LES geometry and mesh overview for the pulsed computations of the diaphragm. 


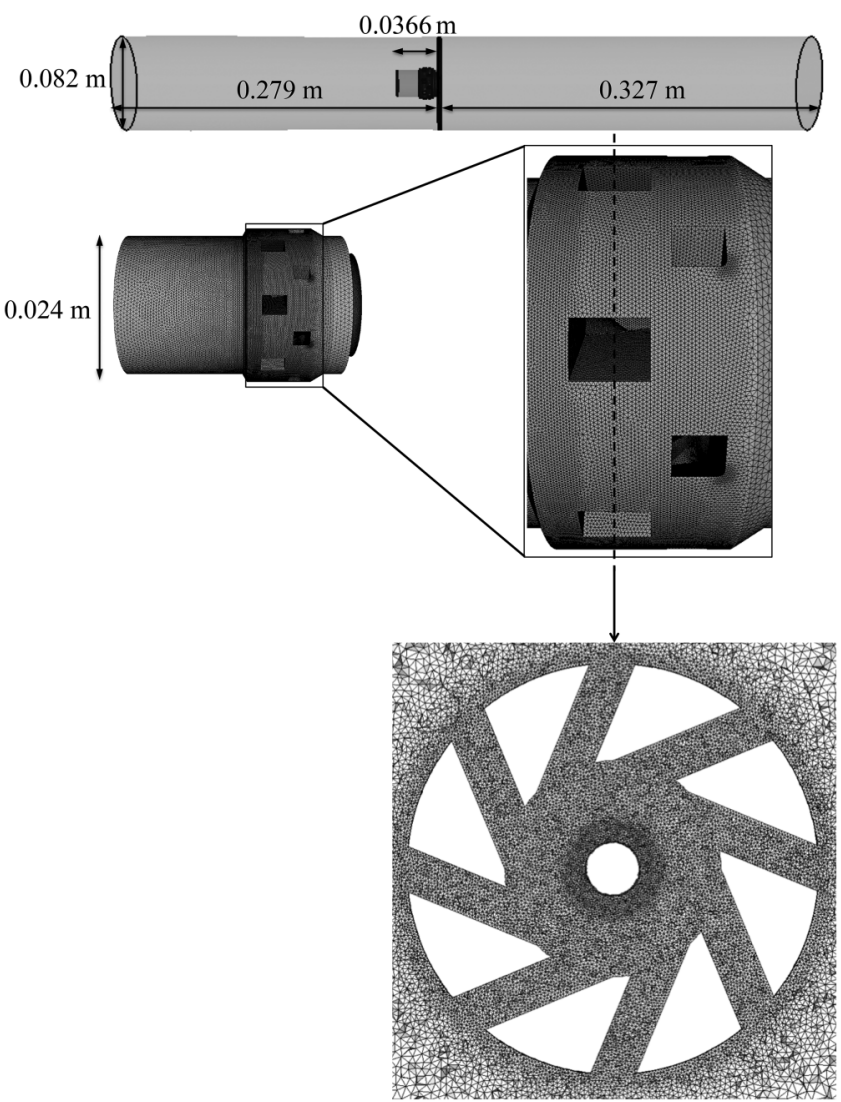

Fig. 11 LES and mesh overview for the pulsed computations of the swirler.

constructing the $\mathcal{E}(f)$ curve of Fig. 8, which is not practical in LES. Therefore, a different method is used to obtain the dissipation rates from LES: the acoustic-mode-triggering (AMT) approach [70].

The idea of the AMT is to superimpose a given acoustic mode (computed from a Helmholtz solver, say) to the mean flowfields computed by a nondisturbed LES [70]. The resulting disturbed solution is used to initialize a new LES computation, in which the initial acoustic perturbations are damped by the presence of the diaphragm or swirler. No forcing is applied. The acoustic system is simply initially displaced from equilibrium. As for a damped oscillator, this results in decaying acoustic oscillations, whose decay rate and frequency are captured by the LES (Fig. 12).

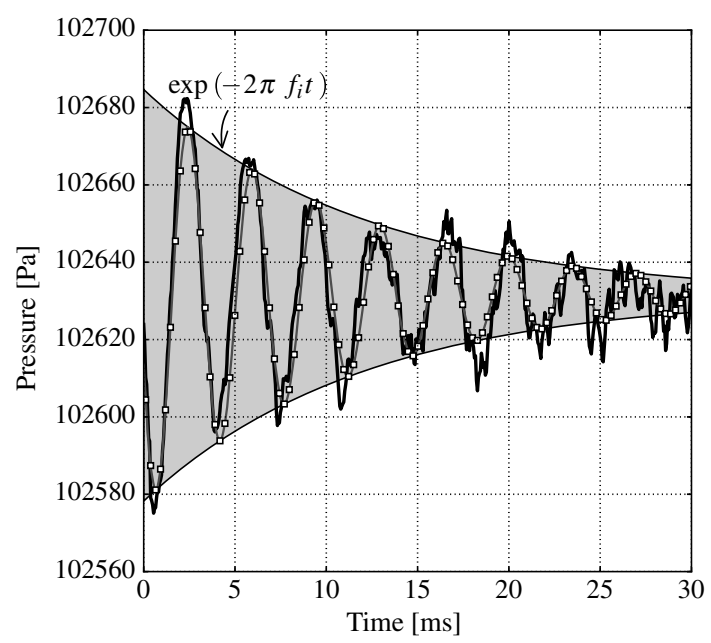

The geometries used in the AMT computations are basically the same as those used for the pulsed computations (Figs. 10 and 11), but the duct lengths are modified to match the experimental duct lengths, plus the end corrections. The mesh refinement is the same as for the pulsed computations. A velocity-imposed, fully reflective boundary condition is enforced at the inlet. For the outlet, a pressure-imposed characteristic condition with relaxation toward a target value was tuned to obtain a reflection coefficient with radiation losses [71] $R_{\mathrm{rad}}(\omega)=-\left[1 / 4(k a)^{2}-1\right] /\left[1 / 4(k a)^{2}+1\right]$ (with $a$ the pipe radius). The formulation of the outlet boundary condition is an extension of the one described in the work of Selle et al. [61]. With their notation, the incoming wave amplitude $\mathcal{L}_{1}$ is set to $\overline{\mathcal{L}}_{1}=K\left(P-P_{\infty}\right)-R_{K} \mathcal{L}_{5}$, and the associated reflection coefficient is (Fig. 13)

$$
R_{\mathrm{LES}}(\omega)=-R_{K}-\frac{1-R_{K}}{1-(2 i \omega / K)}
$$

with $K$ the relaxation coefficient and $R_{K}$ the value of the reflection coefficient when $K \rightarrow 0$. A cut-off frequency can be defined as $f_{c}=K / 4 \pi$, at which the phase is equal to $\pi+\phi$ with $\phi=\tan ^{-1}\left[\left(1-R_{K}\right) /\left(1+R_{K}\right)\right]$. For the diaphragm, $K$ is fixed to ensure no pressure drift, whereas $R_{K}$ is adjusted to set $\left|R_{\mathrm{LES}}\right|\left(\omega_{0}\right)=$ $\left|R_{\text {rad }}\left(\omega_{0}\right)\right|$ at the desired angular frequency $\omega_{0}$ (Table 3 ). For the swirler, $K$ is fixed to the highest value allowed in the LES code to obtain a fully reflective boundary, as will be recalled later.

To measure the real frequency and damping rate, the dynamic mode decomposition (DMD) [72] is performed on the pressure and velocity signals measured at upstream and downstream probes. The DMD is able to isolate the frequency of the excited mode and its decay rate. Figure 12 shows an example of such a filtering for a probe
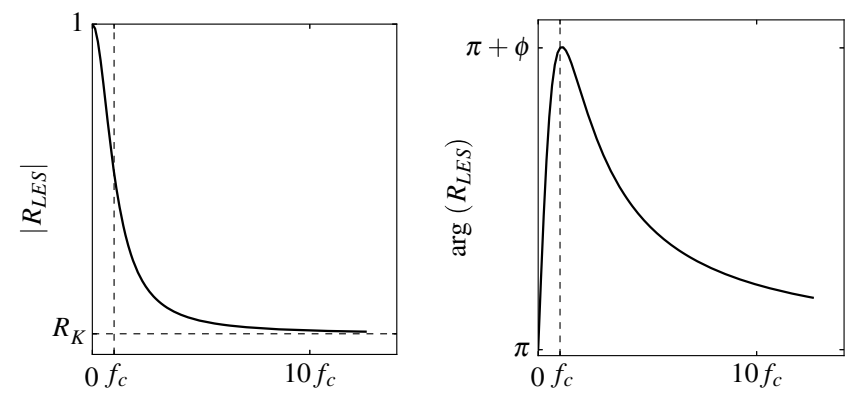

Fig. 13 Modulus (left) and phase (right) of the AMT outlet reflection coefficient $\boldsymbol{R}_{\mathrm{LES}}$.

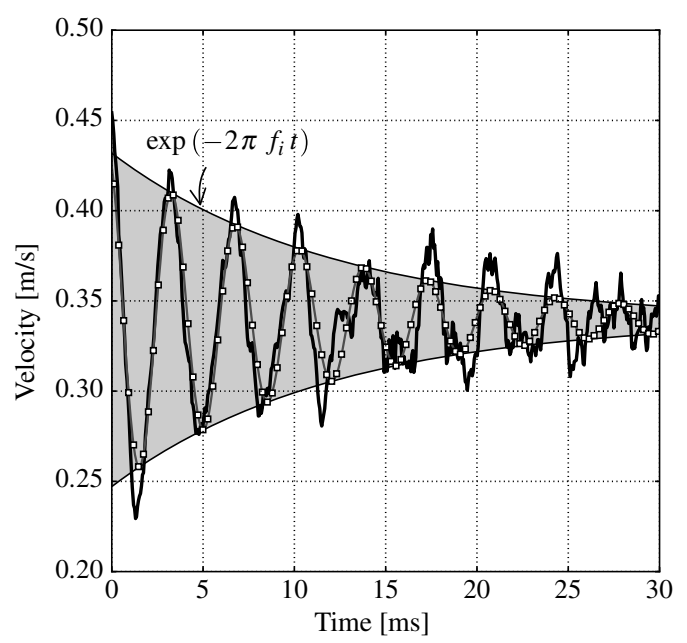

\section{- LES}

$\square$ DMD - reconstruction at $\mathrm{f}=284.9-13.2 \mathrm{j} \mathrm{Hz}$

Fig. 12 DMD reconstruction of the pressure (left) and velocity (right) signal at a probe. 
Table 3 Reflection coefficient $R_{\text {IFS }}$ effectively measured in the diaphragm AMT computations vs theoretical values with radiation losses $R_{\text {rad }}$

\begin{tabular}{lccc}
\hline \hline Frequency, $\mathrm{Hz}$ & $R_{\text {rad }}$ & $\left|R_{\mathrm{LES}}\right|$ & $\arg \left(R_{\mathrm{LES}}\right), \mathrm{rad}$ \\
\hline 77 & -0.9984 & 1.0063 & 3.1408 \\
269 & -0.9803 & 0.9847 & 3.1413 \\
387 & -0.9596 & 0.9597 & 3.1727 \\
604 & -0.9039 & 0.9029 & 3.1551 \\
757 & -0.8534 & 0.8500 & 3.1438 \\
894 & -0.8012 & 0.8022 & 3.1414 \\
\hline \hline
\end{tabular}

signal in the AMT computation of the swirler $(U=0.34 \mathrm{~m} / \mathrm{s}$ and $f=(284.9-13.2 \mathrm{i}) \mathrm{Hz})$. DMD also provides the amplitude and phase of the acoustic fluctuations for each frequency. This information could be used directly to reconstruct the eigenmode, but an additional least-squares fit was performed to determine the acoustic quantities as a sum of plane-wave amplitudes, as done for the experiments in Sec. III.B.1. The pressure signals at LES probes are used to construct system (6) that is inverted using Eq. (7). This procedure smoothes the acoustic fields and helps separate them from noise or hydrodynamic fluctuations (Fig. 14).

\section{Helmholtz-Solver Eigenmodes and Eigenfrequencies with MBC Boundaries}

Once the experimental (Sec. III.B.2) and LES (Sec. III.C.1) matrices are measured, they can be used in the Helmholtz solver with MBC. To use the MBC, the setup geometry has to be modified. As explained in Sec. II, the acoustic element is not meshed anymore. Instead, the upstream and downstream tubes are cut so as to remove completely the acoustic element. The matrices constructed in Secs. III.B.2 and III.C.1 are used to represent adequately the transition between up- and downstream cuts (Fig. 15b).

At the test-rig ends, the boundary conditions are similar for both swirler and diaphragm cases (Fig. 15b). The inlet and outlet patches are defined, respectively, as a $u^{\prime}=\overline{0}$ boundary condition with an end correction of $\delta_{\text {in }}=1 \mathrm{~cm}$ and a $p^{\prime}=0$ boundary condition with an end correction of $\delta_{\text {out }}=2.5 \mathrm{~cm}$ (see Sec. III.A). With $k=\omega / c$ being the wave number, the inlet and outlet reduced impedances $\hat{p} /\left(\rho_{o} c_{o} \hat{u}\right)$ are then

$$
\begin{gathered}
Z_{\text {in }}=\frac{e^{2 i k \delta_{\text {in }}}+1}{e^{2 i k \delta_{\text {in }}}-1} \\
Z_{\text {out }}=\frac{e^{2 i k \delta_{\text {out }}}-1}{e^{2 i k \delta_{\text {out }}}+1}-\frac{1}{4} \alpha(k a)^{2}
\end{gathered}
$$
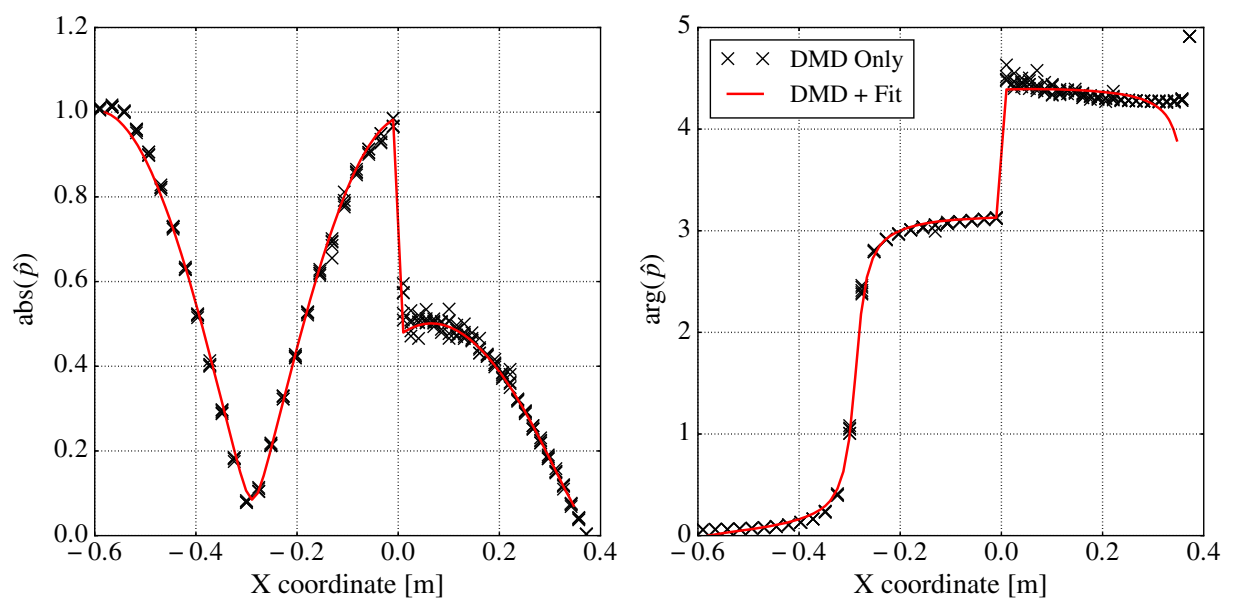

Fig. 14 Plane waves fit of an acoustic mode (swirler case at $U=0.34 \mathrm{~m} / \mathrm{s}, f=(284.9-13.2 i) \mathrm{Hz}$ ).
When comparing the Helmholtz eigenmodes with experiments or LES runs with a partially reflective outlet (C2-EXP and C2-LES in Fig. 5), $\alpha$ is set to 1 , and the term $-\left(1 / 4 k a^{2}\right)$ accounts for radiation losses [71]. For the diaphragm study, $\alpha$ is therefore fixed to 1 . In the swirler case, Helmholtz solutions are compared only to the LES data with a fully reflective outlet, so that no radiation loss is added in the Helmholtz computation $(\alpha=0)$.

The mean thermodynamic properties are the same as the experimental ones, given in Sec. III.A.

Additionally, Helmholtz computations on the complete geometry (i.e., with the same duct lengths as the experimental setup and with a discretized diaphragm/swirler; see Fig. 15a for the diaphragm case) are performed for two reasons: 1) to get acoustic fluctuations for LES eigenmode runs with the AMT approach, and 2) to compare the result of a conventional Helmholtz solution (i.e., assuming the Helmholtz equation holds within the diaphragm and the swirler) with the one obtained, thanks to the MBC methodology in comparisons C2-LES and C2-EXP (Fig. 5).

The meshes for the complete geometries and MBC geometries are optimized for the Helmholtz solver, and are consequently coarser and more uniform than the LES ones. All but one mesh contain around 100,000 nodes and 500,000 cells, with a typical cell size of $3.6 \mathrm{~mm}$. The exception is the complete swirler mesh, which contains 300,000 nodes and 1,500,000 cells necessary to discretize the fine swirler vanes. The typical cell size for the latter mesh varies from $1 \mathrm{~mm}$ in the vanes to $8.2 \mathrm{~mm}$ in the pipes.

\section{Application to a Diaphragm}

The methodology is first validated on the diaphragm configuration. It was checked that the chosen boundary conditions provide values of eigenfrequencies close to the experiment when computing the complete geometry with the Helmholtz solver; see Table 4. Taking into account the radiation losses at the outlet only introduces a small damping rate. For the first mode, however, a $10 \mathrm{~Hz}$ discrepancy is observed. This is due to acoustic coupling between the test rig and the loudspeaker casing. The coupling introduces a velocity discontinuity that is stronger for frequencies close to the Helmholtz resonance frequency of the loudspeaker cavity $(\approx 75 \mathrm{~Hz})$, as is the case for the first main rig eigenmode. For higher-order modes, the coupling between the rig and the loudspeaker cavity is negligible. It was checked but not shown here that the first eigenfrequency is better predicted (around $90 \mathrm{~Hz}$ ) when including the loudspeaker casing in the Helmholtz geometry. However, to simplify the LES and Helmholtz computations, we decided to remove the loudspeaker casing from all geometries, keeping in mind that this makes comparison with the first experimental mode inappropriate.

For the diaphragm case, the quality of the results and the subsequent conclusions are the same for $U=0.17 \mathrm{~m} / \mathrm{s}$ and $U=0.34 \mathrm{~m} / \mathrm{s}$. Therefore, all the results shown here are valid for $U=0.34 \mathrm{~m} / \mathrm{s}$ if 

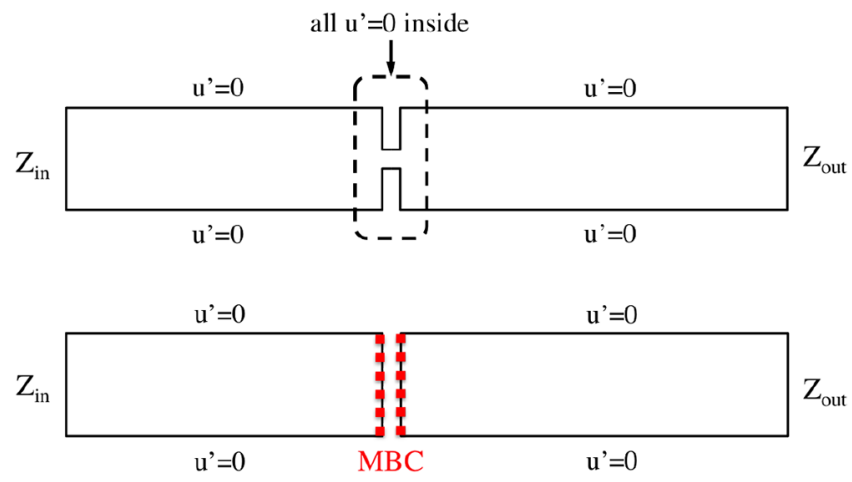

Fig. 15 Boundary conditions for a) standard Helmholtz (complete geometry) and b) MBC Helmholtz computations.

nothing is mentioned. The scattering-matrix coefficients acquired experimentally for the diaphragm of Fig. 6a, as well as the values obtained numerically, are plotted in Fig. 16. The agreement between experimental, numerical, and theoretical (Howe's model [28]) results is good (comparison $\mathrm{C} 1$ in Fig. 5): the numerical coefficients match almost exactly the experimental ones, confirming that LES is suitable to compute acoustic matrices. (The matrix associated to Howe's model is defined as $S(\omega)=1 /[1-\xi(\omega)]\left[\begin{array}{cc}1 & -\xi(\omega) \\ -\xi(\omega) & 1\end{array}\right]$ with $\xi(\omega)=$ $i \omega d^{2} / 2 c_{0} K_{R}$ for a plate of size $d$ and Rayleigh conductivity $K_{R}$.)

The $S$ matrix already contains information relevant to dissipation. For example, an acoustic-flux-balance criterion [73] based on the eigenvalues $\lambda_{\min }, \lambda_{\max }$ of the real-valued matrix $\mathbb{I}-\bar{S}^{*} \boldsymbol{S}$, with $\boldsymbol{S}^{*}$ the Hermitian form (complex conjugate transpose) of $\boldsymbol{S}$ states that

1) If $\lambda_{\min }>0$ and $\lambda_{\max }>0, S$ dissipates acoustic energy.

2) If $\lambda_{\min }<0$ and $\lambda_{\max }<0, S$ produces acoustic energy.

3) If $\lambda_{\min }<0$ and $\lambda_{\max }>0, S$ can produce or dissipate acoustic energy. Nothing can be said a priori. The sign of the acoustic flux balance can change, depending on the inlet acoustic variables, which are not known before connecting the matrix to the Helmholtz solver.

Figure 17 confirms the dissipative behavior of experimental and numerical matrices, although numerical matrices seem to dissipate slightly less than experimental matrices. For reference, the criterion is also computed for Howe's model in Fig. 17. (The eigenvalues $\lambda_{\min }$, $\lambda_{\max }$ can be computed analytically for Howe's model as $\lambda_{\min }(\omega)=0$ and $\lambda_{\max }(\omega)=-\left\{4 \Re[\xi(\omega)] /|1-\xi(\omega)|^{2}\right\}$. The quantity $\Re[\xi(\omega)]=$ $-\left\{\omega d^{2} \Delta(\omega) / 4 c_{0} a\left[\Gamma(\omega)^{2}+\Delta(\omega)^{2}\right]\right\}$ depends on the real-valued functions $\Gamma(\omega)$ and $\Delta(\omega)$ defined in [28]. Because $\Delta(\omega)$ is positive for all $\omega, \lambda_{\max }(\omega)$ is always positive. According to Howe's model, the perforated plate always dissipates acoustic energy.)

This dissipative behavior is fairly well captured in the eigenfrequencies computed with Helmholtz-MBC, compared to the ones measured in the experiment and in the LES (Fig. 18). The exception is the first experimental mode, as expected and explained earlier. The agreement is much better when comparing MBC with LES, because the LES and Helmholtz-solver geometries were the same in this case. Compared to the standard Helmholtz approach, the introduction of MBC improves the damping-rate prediction for all modes computed. It also provides the correct evolution of the eigenfrequencies with a flow-rate increase with both the matrices

Table 4 Eigenfrequencies (in $\mathrm{Hz}$ ) of the diaphragm case at $U=0 \mathrm{~m} / \mathrm{s}$ (experiments and standard Helmholtz computations with and without radiation losses)

\begin{tabular}{lcc}
\hline \hline Experiment & Standard Helmholtz & Standard Helmholtz with end losses \\
\hline 87.5 & $78.1-0.0 i$ & $78.1-0.0 i$ \\
267.2 & $268.1-0.0 i$ & $268.1-0.8 i$ \\
372.3 & $375.4-0.0 i$ & $375.4-1.0 i$ \\
605.2 & $604.9-0.0 i$ & $604.9-0.6 i$ \\
751.4 & $760.1-0.0 i$ & $759.8-9.7 i$ \\
898.7 & $897.0-0.0 i$ & $896.7-0.9 i$ \\
\hline \hline
\end{tabular}
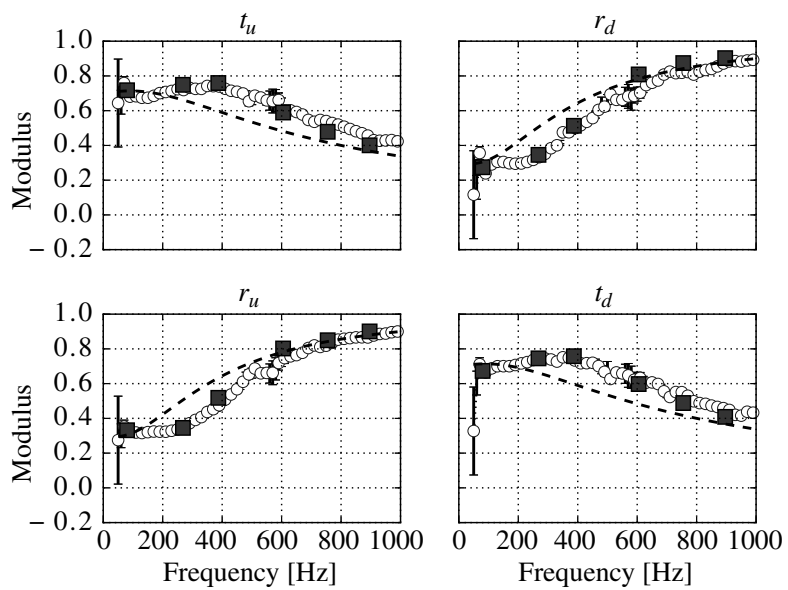

a) Moduli of the 2-port matrix for the diaphragm at $U=0.34 \mathrm{~m} / \mathrm{s}$
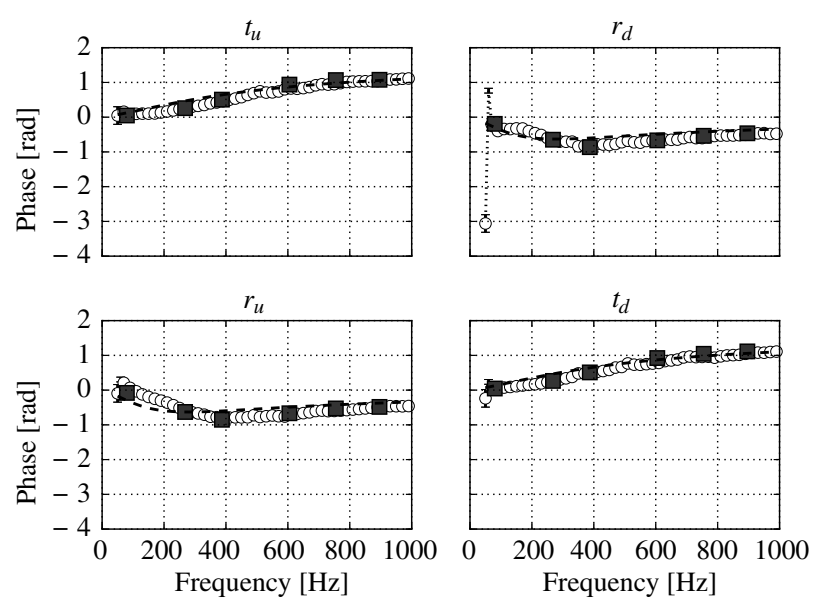

b) Phases of the 2-port matrix for the diaphragm at $U=0.34 \mathrm{~m} / \mathrm{s}$

- $\square$ LES --- Howe model

Fig. 16 Diaphragm case at $U=0.34 \mathrm{~m} / \mathrm{s}$; scattering matrix from experiments, LES and analytical model.

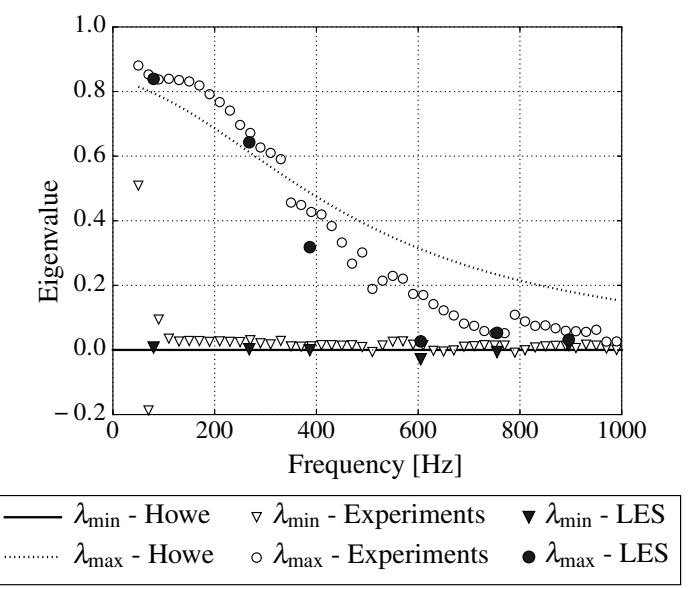

Fig. 17 Diaphragm case at $U=0.34 \mathrm{~m} / \mathrm{s}$; Eigenvalues of $\mathbb{I}-S^{*} S$ in experiments, LES and analytical model.

from experiments and from LES (comparison C3 in Fig. 5), as shown for the first four modes in Figs. 19 and 20.

To further evaluate the impact of the dissipative diaphragm on the surrounding acoustics, the structure of the second eigenmode is now 


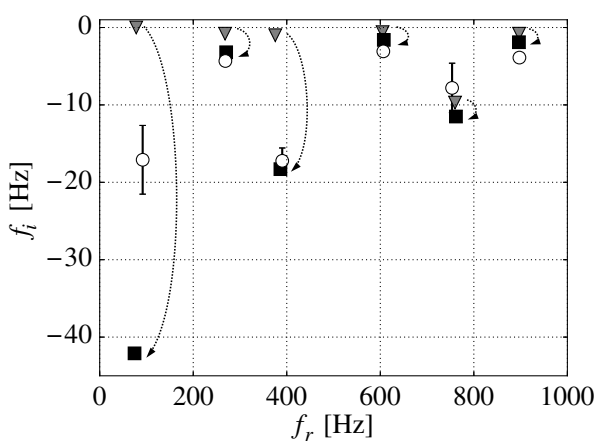

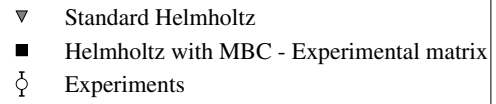

a) Helmholtz solver with MBC vs experiments (C2-EXP in Fig. 5)

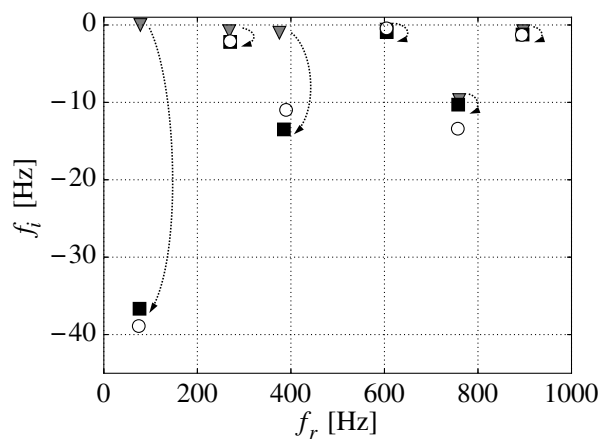

$\nabla \quad$ Standard Helmholtz

- Helmholtz with MBC - LES matrix - LES

b) Helmholtz solver with MBC vs LES (C2-LES in Fig. 5)

Fig. 18 Diaphragm case. Eigenfrequencies from standard and MBC Helmholtz vs a) experiments, b) LES.
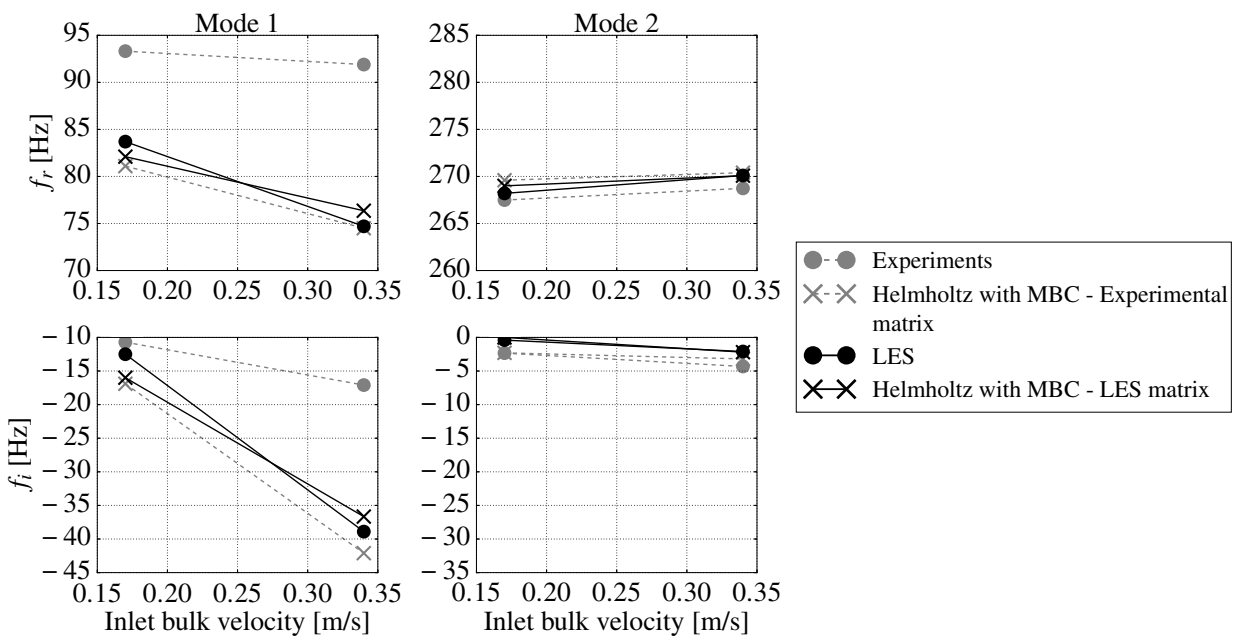

Fig. 19 Diaphragm case. Eigenfrequencies vs $U$ (top: real part, bottom; imaginary part).
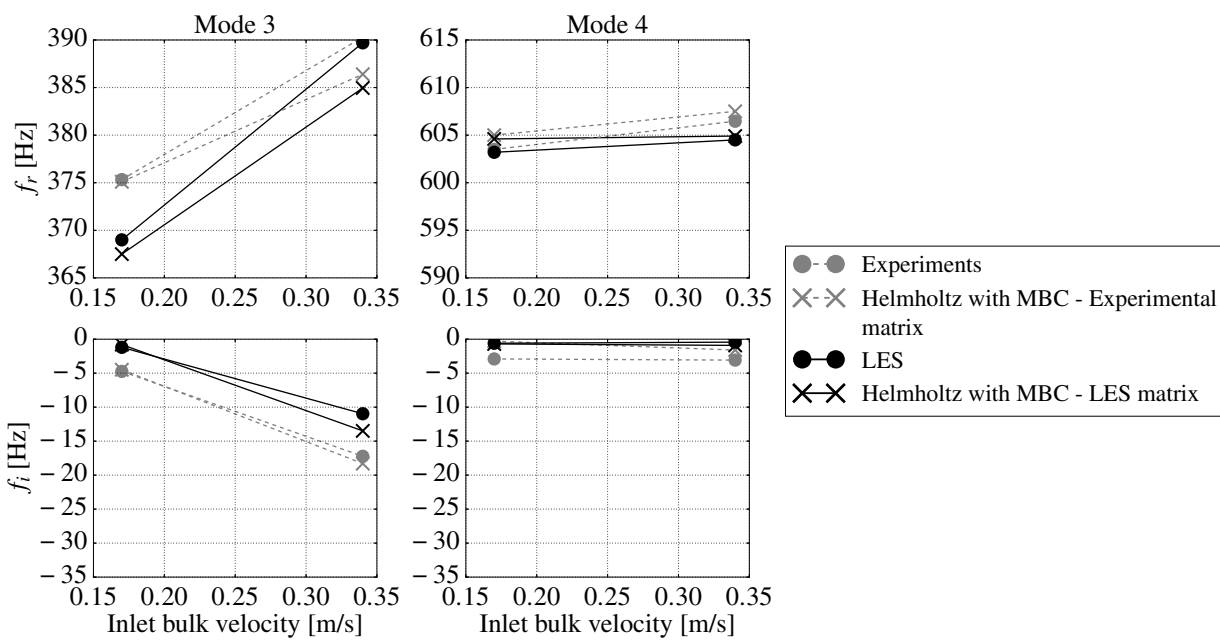

Fig. 20 Diaphragm case. Eigenfrequencies vs $U$, continued (top: real part, bottom: imaginary part).

compared between experiments, LES, and Helmholtz-solver computations. This is done by plotting the modulus and phase of $\hat{p}$, normalized at $x=-0.58 \mathrm{~m}$. Both experiments (Fig. 21) and LES
(Fig. 22) show that the presence of a mean flow through the diaphragm modifies the pressure jump across the orifice, which is a direct indication of acoustic losses. This effect is captured by the 

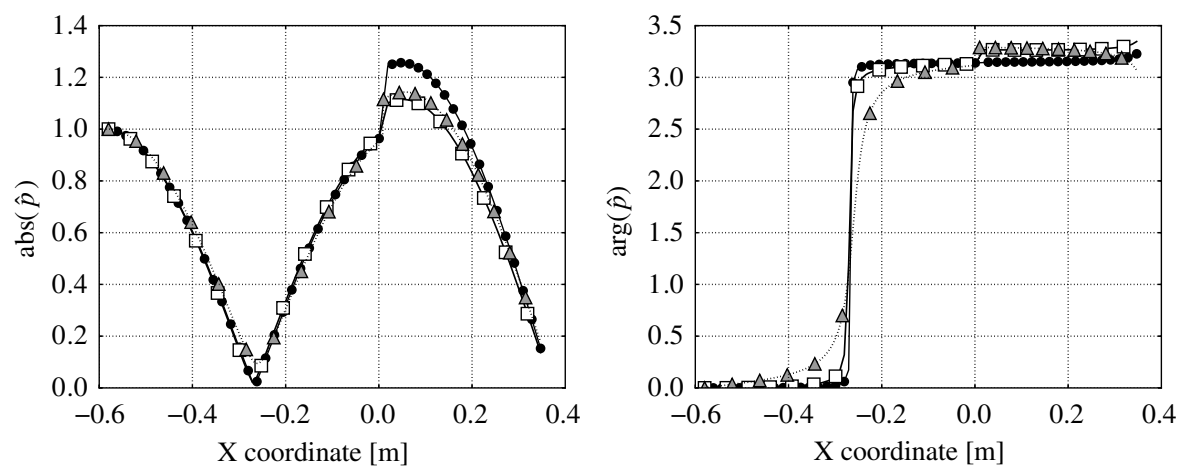

$\bullet$ Standard Helmholtz $\quad \square \square \square \begin{aligned} & \text { Helmholtz with MBC } \\ & \text { (Experimental matrix) }\end{aligned} \quad \triangle \cdots \cdots \cdot \triangle$ Experiment

Fig. 21 Diaphragm case. Second eigenmode structure from experiments, standard and MBC Helmholtz computations.
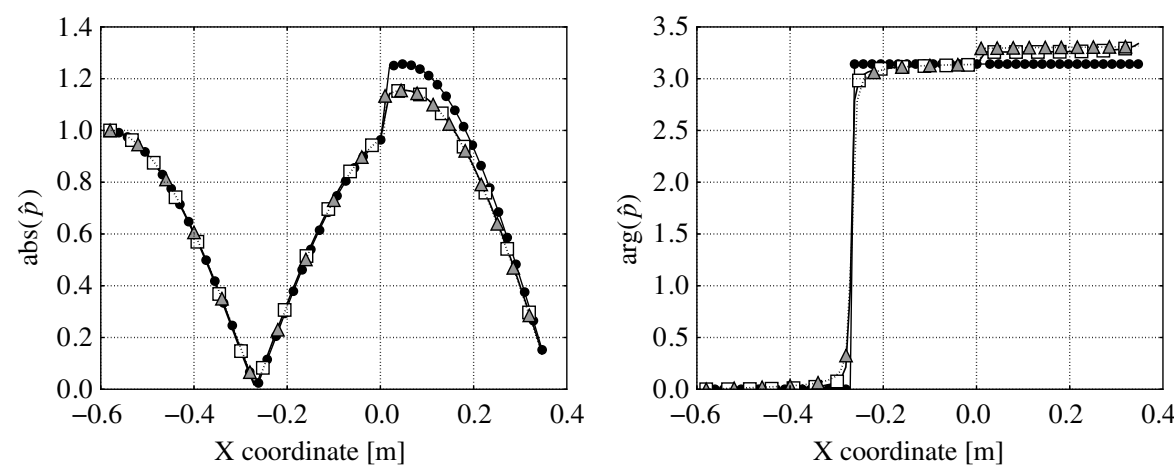

$\longrightarrow$ Standard Helmholtz

$\square \square$

Helmholtz with MBC

(LES matrix)

$\triangle \cdots \cdots \cdot \triangle$ LES

Fig. 22 Diaphragm case. Third mode structure from LES, standard and MBC Helmholtz computations.

Helmholtz solver with MBC, but not by a standard Helmholtz computation (Figs. 21 and 22). These good results support our initial assumption that, at first order, the impact of mean flow is important only at locations where damping through vortex shedding is present, and can be neglected elsewhere, so that acoustics are well represented by the zero-mean-flow Helmholtz equation (1).

\section{Application to an Industrial Swirler}

In the diaphragm configuration, the flow was laminar and fairly simple: the only complexity was the shear layer created at the downstream lips of the diaphragm. Moreover, the analytical model of Howe [28] was available for comparison. Now, the methodology is applied to the more challenging swirler element (Fig. 6b) for which there is no theoretical formula. Downstream of the swirler, the flow features complex phenomena, such as recirculation zones or precessing vortex cores. Because the comparison with the LES (C2-LES in Fig. 5) gave the best results for the diaphragm, it is the only comparison performed for the swirler. The LES computations were performed for the highest bulk velocity $U=$ $0.34 \mathrm{~m} / \mathrm{s}$ to maximize the acoustic-hydrodynamic coupling. Compared to the diaphragm case, the outlet is fully reflective both in the LES and in the Helmholtz solver. Two modes are studied here: mode A and mode B with frequencies around 285 and $590 \mathrm{~Hz}$, respectively, at $U=0.34 \mathrm{~m} / \mathrm{s}$ (as assessed by LES, Table 5). The swirler matrix coefficients were computed at 280, 290, 370, and $590 \mathrm{~Hz}$, and are listed in Table 6, and linear interpolation is used to obtain the effective coefficients at the Helmholtz-solver frequency. With the exception of $370 \mathrm{~Hz}$, which is used later to assess the error made if the matrix is not measured at the correct frequency, the other frequencies were chosen close to the frequencies of mode $\mathrm{A}$ and mode $B$.
In Table 5, the results of the Helmholtz solver are compared to the LES for mode A and mode B. In terms of damping rates, the MBC methodology captures the correct order of magnitude, contrary to the standard Helmholtz solver, which leads to $\omega_{i}=0$, as expected. The relative error on the frequencies, computed as $\left|f_{\text {Helmholtz }}-f_{\text {LES }} / f_{\text {LES }}\right|$, remains around $1 \%$ for both modes.

Table 5 Complex frequencies (in $\mathrm{Hz}$ ) of mode $\mathrm{A}$ and mode $\mathrm{B}$ with the swirler at $U=0.34 \mathrm{~m} / \mathrm{s}$, from LES, Helmholtz solver with LES matrix, and standard Helmholtz solver

\begin{tabular}{lcc}
\hline \hline & Mode A & Mode B \\
\hline Standard Helmholtz & $317.4-0.0 i$ & $591.2-0.0 i$ \\
LES & $284.9-13.2 i$ & $589.1-3.2 i$ \\
Helmholtz with LES matrix & $287.5-13.7 i$ & $597.7-1.3 i$ \\
Relative error, \% & 0.9 & 1.5 \\
\hline \hline
\end{tabular}

The relative difference between Helmholtz and LES results is also displayed.

Table 6 Swirler matrix

\begin{tabular}{lcccc}
\hline \hline & $280 \mathrm{~Hz}$ & $290 \mathrm{~Hz}$ & $370 \mathrm{~Hz}$ & $590 \mathrm{~Hz}$ \\
\hline$t_{u}$ & $0.189+0.002$ & $0.268-0.054 j$ & $0.465+0.043 j$ & $0.043+0.117 j$ \\
$r_{d}$ & $0.833+0.099 j$ & $0.775+0.076 j$ & $0.568+0.203 j$ & $0.946+0.012 j$ \\
$r_{u}$ & $0.806+0.079 j$ & $0.712+0.094 j$ & $0.538+0.033 j$ & $0.955+0.001 j$ \\
$t_{d}$ & $0.170-0.014 j$ & $0.217+0.005 j$ & $0.392-0.079 j$ & $0.034+0.196 j$ \\
$\lambda_{\min }$ & -0.006 & 0.016 & 0.003 & -0.038 \\
$\lambda_{\max }$ & 0.582 & 0.741 & 0.965 & 0.176 \\
\hline \hline
\end{tabular}

Scattering-matrix coefficients from LES for $U=0.34 \mathrm{~m} / \mathrm{s}$ and eigenvalues of $\mathbb{I}-\boldsymbol{S}^{*} \boldsymbol{S}$. 

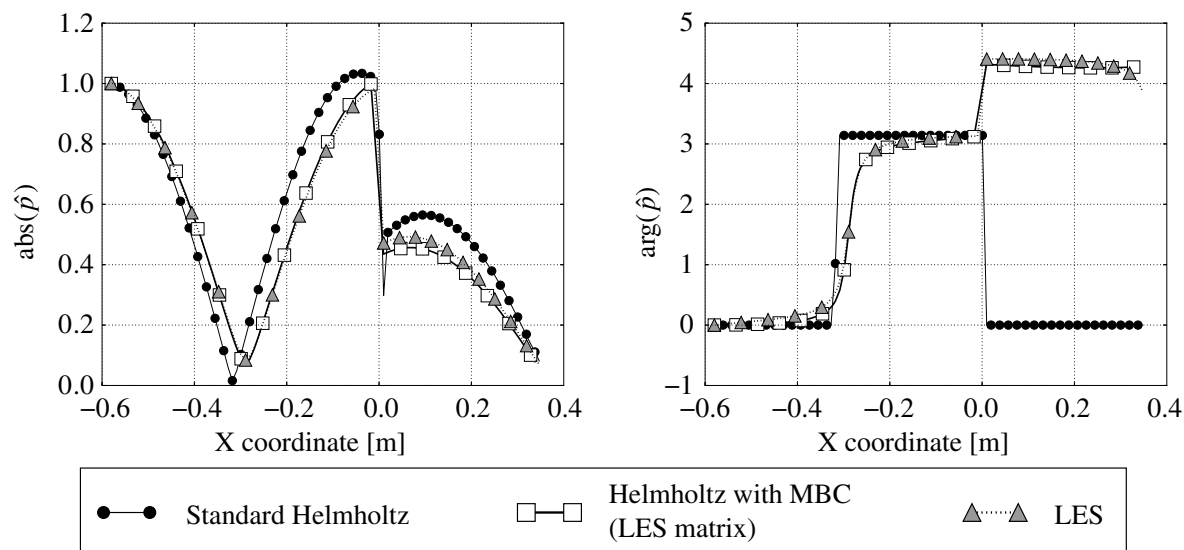

Fig. 23 Swirler case. Structure of mode A from LES, standard and MBC Helmholtz computations.
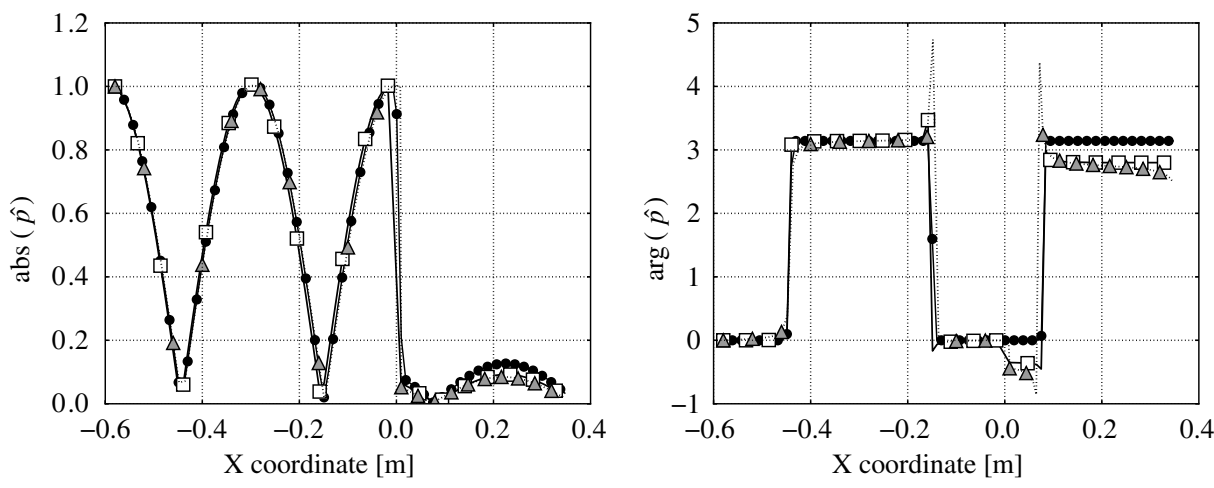

$\bullet$ Standard Helmholtz

Helmholtz with MBC

(LES matrix)

$\triangle \cdots \cdot \Delta$ LES

Fig. 24 Swirler case. Structure of mode B from LES, standard and MBC Helmholtz computations.

The eigenmode structures are also well retrieved by the MBC approach, as illustrated in Figs. 23 and 24.

In a general case with turbulent complex flow, as is the case with the swirler, it can be difficult to properly separate acoustic fluctuations from hydrodynamic ones. This results in uncertainties in the acoustic matrix coefficients. Moreover, the frequencies of the modes of interest are not always available beforehand. In this case, the matrix can be measured for a chosen set of frequencies, and linear interpolation is applied to retrieve the coefficients at the desired frequency, as was done here. This introduces an additional uncertainty. To assess the effect of these uncertainties on the result of the MBC-Helmholtz methodology, 50 additional Helmholtz computations are performed for mode A by varying the coefficients of the scattering matrix as follows. Each coefficient can have five different values: the nominal value, the nominal value plus $\Delta_{S}$, the nominal value minus $\Delta_{S}$, the nominal value plus $j \Delta_{S}$, and the nominal value minus $j \Delta_{S}$. The total number of matrix combinations amounts to 695 , among which 50 combinations are sampled randomly. $\Delta_{S}$ is a real number chosen arbitrarily to 0.03 , and accounts for the uncertainty on the matrix due to the measurement method, but also on the frequency at which measurements are taken. Variations of the order of $\Delta_{S}$ on the scattering matrix were observed when interpolating the matrix between 280 and $290 \mathrm{~Hz}$ instead of 280 and $370 \mathrm{~Hz}$ (Table 6).

Figure 25 shows that the complex eigenfrequency computed with $\mathrm{MBC-H}$ elmholtz is indeed sensitive to the value of the matrix coefficients (the nominal result is recalled in Fig. 25 as a white square). In particular, the variations of the imaginary part of the frequency can reach up to $3.5 \mathrm{~Hz}$, corresponding to approximately

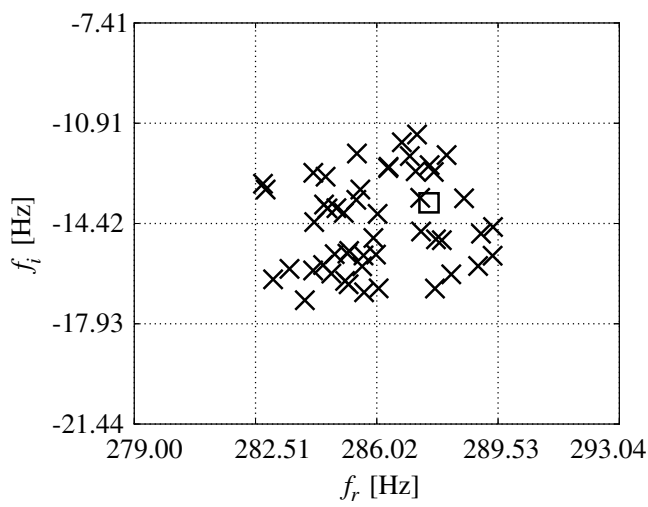

Fig. 25 Swirler case. Sensitivity of the frequency of mode A to matrix coefficients.

$25 \%$ of the nominal value. The eigenmode structure is also greatly impacted (Fig. 26). In Fig. 26, the normalization is different from that used in Fig. 23 , because fixing $\hat{p}=1$ at $x=-0.58 \mathrm{~m}$ would force all modes to collapse in the upstream section. To better see the changes in both upstream and downstream mode structures, $\hat{p}$ is fixed to 1 at $x=0$, the matrix location. The great dispersion of mode shapes around the nominal result in black suggests that the matrices should be carefully measured to obtain satisfying results with the MBC methodology. In our experience, the biggest source 

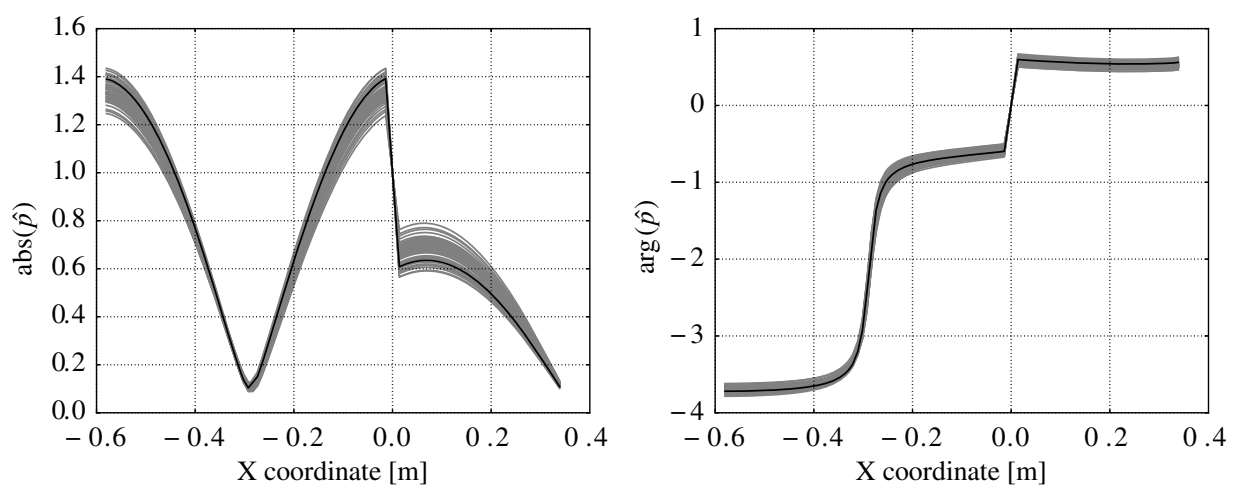

Fig. 26 Swirler case. Sensitivity of the structure of mode A to matrix coefficients.

of error is the frequency of measurement, which should ideally be chosen as close as possible to the frequency of the mode found by AVSP.

\section{Conclusions}

A methodology was presented to describe the acoustic behavior of non-purely acoustic elements in a zero-mean-flow Helmholtz solver. Compared to other approaches, such as LNSE, it has the advantage of being slightly faster and compatible with the prediction of thermoacoustic instabilities (although no instability was investigated in this paper). Here, the elements of interest are represented by their two-port matrix, introduced in the Helmholtz solver, thanks to an MBC. The methodology was applied on an academic configuration to retrieve the dissipative effects of two elements: a diaphragm and a swirler. For these elements, the acoustic-hydrodynamic interaction was the source of important damping rates and changes in the eigenmode structures. These modifications were correctly captured by the Helmholtz solver with MBC, with matrices measured numerically and experimentally, whereas the standard Helmholtz solution misses them. However, a simple sensitivity analysis showed that the matrix should be carefully measured to obtain meaningful results. In particular, the frequency at which the matrix is measured should be as close as possible to the frequency of the mode of interest.

\section{Appendix A: Howe's Model for the Acoustic Damping of a Perforated Plate}

Howe [28] derived a model to account for the acoustic damping created by a perforated plate. Across the plate, the acoustic pressure $\hat{p}$ is discontinuous, whereas the acoustic volume-flow rate $\hat{G}=d^{2} \hat{\boldsymbol{u}}$. $\boldsymbol{n}$ is conserved; $d$ is the average distance between perforations, see Fig. A1. This creates a difference between the inlet acoustic flux $1 / 2 \Re\left(\hat{p}^{+} \hat{G}^{*}\right)$ and the outlet acoustic flux $1 / 2 \Re\left(\hat{p}^{-} \hat{G}^{*}\right)$, which corresponds to the flux dissipated at the plate. The flux difference is directly controlled by a quantity known as the Rayleigh conductivity [32]. The Rayleigh conductivity $K_{R}$ quantifies the ratio of the acoustic-pressure jump over the acoustic volume-flow rate [4], and was derived analytically by Howe [28] for perforated plates. For the plate of Fig. A1

$$
K_{R}=\frac{i \rho_{o} \omega \hat{G}}{\left[\hat{p}^{+}-\hat{p}^{-}\right]}=2 a\left(\Gamma_{S r}-i \Delta_{S r}\right)
$$

in which $a$ is the radius of the perforations (assumed of cylindrical shape), and $\Gamma_{S r}$ and $\Delta_{S r}$ are two real valued functions of the Strouhal number given in [28]. The Strouhal number is defined with the orifice bias speed as $S r=\omega a / U_{\text {ori }}$.

Howe's model can also be used to compute the Rayleigh conductivity of one perforation in a circular plate. In this case, the average distance between perforations $d$ is replaced by $\sqrt{\pi} R$, with $R$ the radius of the plate.

\section{Appendix B: Numerical Parameters for LES Computations}

All CFD computations are performed with the LES solver AVBP [60], codeveloped by CERFACS and IFPEN. This tool is widely used and has been validated in numerous situations $[15,18,30,61-64]$. The numerical scheme two-step Taylor-Galerkin $\mathrm{C}$ is third-order accurate in time and space, and was specifically designed to properly represent unsteady compressible flows with or without combustion [65]. The $\sigma$ model $[66,67]$ is used to model the subgrid-scale stress tensor accurately.

For all computations, the diaphragm and swirler parts are modeled as adiabatic no-slip walls, whereas the duct walls are represented with adiabatic slip conditions. The acoustic losses due to shear stress or thermal diffusion in the main duct are therefore neglected. As said in the Introduction, this is because this effect is small compared to acoustic-hydrodynamic damping in the low-frequency range.

Characteristic Navier-Stokes inlet and outlet boundary conditions [68] are used for pulsed computations. For AMT computations, the inlet and outlet are switched to completely reflecting boundaries, so as to measure the damping rate associated with the diaphragm or swirler, and not the one due to losses at boundaries.

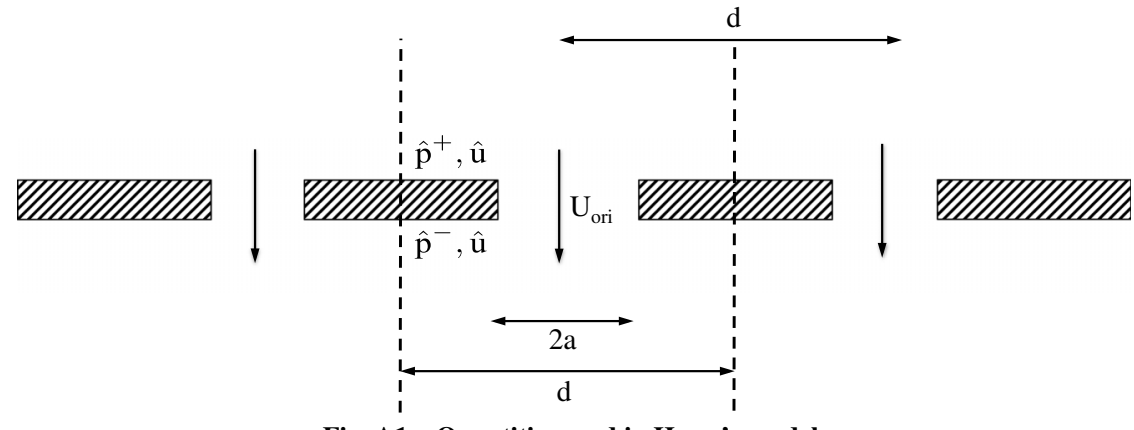

Fig. A1 Quantities used in Howe's model. 


\section{Acknowledgments}

The authors wish to thank A. Misdariis for performing the stationary large-eddy-simulation computations used as a starting point for this analysis, and M. Bauerheim for the fruitful discussions about acoustic-mode triggering. The experiments were performed with the funding from the European Research Council (ERC) under the European Union's Seventh Framework Programme (FP/20072013)/ERC grant agreement ERC-AdG 319067-INTECOCIS. The support from A. Cayre and S. Roux at Safran-Société Nationale d'Etude et de Construction de Moteurs d'Aviation is also gratefully acknowledged.

\section{References}

[1] Candel, S., Durox, D., Schuller, T., Bourgouin, J.-F., and Moeck, J. P., "Dynamics of Swirling Flames," Annual Review of Fluid Mechanics, Vol. 46, No. 1, Jan. 2014, pp. 147-173. doi:10.1146/annurev-fluid-010313-141300

[2] Culick, F. E. C., and Kuentzmann, P., "Unsteady Motions in Combustion Chambers for Propulsion Systems," NATO Research and Technology Organisation AG-AVT-039, Neuilly-sur-Seine, France, 2006.

[3] Lieuwen, T. C., Unsteady Combustor Physics, Cambridge Univ. Press, Cambridge, 2012. doi:10.1017/cbo9781139059961

[4] Rayleigh, L., The Theory of Sound, Macmillan, London, 1894, pp. 226234 (reprinted by Dover, New York, 1945).

[5] Candel, S., "Combustion Dynamics and Control: Progress and Challenges," Proceedings of the Combustion Institute, Vol. 29, No. 1, Jan. 2002, pp. 1-28. doi:10.1016/S1540-7489(02)80007-4

[6] Lieuwen, T., and Yang, V., Combustion Instabilities in Gas Turbine Engines: Operational Experience, Fundamental Mechanisms, and Modeling, Vol. 210, Progress in Astronautics and Aeronautics, AIAA, Reston, VA, 2005.

[7] Evesque, S., and Polifke, W., "Low-Order Acoustic Modelling for Annular Combustors: Validation and Inclusion of Modal Coupling," ASME Turbo Expo 2002: Power for Land, Sea, and Air, ASME International, New York, 2002, pp. 321-331. doi:10.1115/gt2002-30064

[8] Schuermans, B., Bellucci, V., and Paschereit, C. O., "Thermoacoustic Modeling and Control of Multi Burner Combustion Systems," ASME Turbo Expo 2003, ASME International, New York, 2003, pp. 509-519. doi:10.1115/gt2003-38688

[9] Stow, S. R., and Dowling, A. P., "Low-Order Modelling of Thermoacoustic Limit Cycles," ASME Turbo Expo 2004: Power for Land, Sea, and Air, ASME International, New York, 2004, pp. 775-786. doi:10.1115/gt2004-54245

[10] Bauerheim, M., Parmentier, J., Salas, P., Nicoud, F., and Poinsot, T., "An Analytical Model for Azimuthal Thermoacoustic Modes in an Annular Chamber Fed by an Annular Plenum," Combustion and Flame, Vol. 161, No. 5, May 2014, pp. 1374-1389. doi:10.1016/j.combustflame.2013.11.014

[11] Han, X., Li, J., and Morgans, A. S., "Prediction of Combustion Instability Limit Cycle Oscillations by Combining Flame Describing Function Simulations with a Thermoacoustic Network Model," Combustion and Flame, Vol. 162, No. 10, Oct. 2015, pp. 3632-3647. doi:10.1016/j.combustflame.2015.06.020

[12] Huang, Y., Sung, H. G., Hsieh, S. Y., and Yang, V., "Large Eddy Simulation of Combustion Dynamics of Lean-Premixed SwirlStabilized Combustor," Journal of Propulsion and Power, Vol. 19, No. 5, Nov. 2003, pp. 782-794. doi: $10.2514 / 2.6194$

[13] Schmitt, P., Poinsot, T., Schuermans, B., and Geigle, K. P., "Large-Eddy Simulation and Experimental Study of Heat Transfer, Nitric Oxide Emissions and Combustion Instability in a Swirled Turbulent HighPressure Burner," Journal of Fluid Mechanics, Vol. 570, Jan. 2007, pp. 17-46. doi:10.1017/S0022112006003156

[14] Staffelbach, G., Gicquel, L., Boudier, G., and Poinsot, T., "Large Eddy Simulation of Self-Excited Azimuthal Modes in Annular Combustors,' Proceedings of the Combustion Institute, Vol. 32, No. 2, 2009, pp. 2909-2916. doi:10.1016/j.proci.2008.05.033

[15] Wolf, P., Staffelbach, G., Gicquel, L., Muller, J.-D., and Poinsot, T., "Acoustic and Large Eddy Simulation Studies of Azimuthal Modes in Annular Combustion Chambers," Combustion and Flame, Vol. 159,
No. 11, Nov. 2012, pp. 3398-3413.

doi:10.1016/j.combustflame.2012.06.016

[16] Gicquel, L. Y. M., Staffelbach, G., and Poinsot, T., "Large Eddy Simulations of Gaseous Flames in Gas Turbine Combustion Chambers," Progress in Energy and Combustion Science, Vol. 38, No. 6, 2012, pp. 782-817. doi:10.1016/j.pecs.2012.04.004

[17] Hermeth, S., Staffelbach, G., Gicquel, L., and Poinsot, T., "LES Evaluation of the Effects of Equivalence Ratio Fluctuations on the Dynamic Flame Response in a Real Gas Turbine Combustion Chamber," Proceedings of the Combustion Institute, Vol. 34, No. 2, 2013, pp. 3165-3173. doi:10.1016/j.proci.2012.07.013

[18] Ghani, A., Poinsot, T., Gicquel, L., and Staffelbach, G., "LES of Longitudinal and Transverse Self-Excited Combustion Instabilities in a Bluff-Body Stabilized Turbulent Premixed Flame," Combustion and Flame, Vol. 162, No. 11, Nov. 2015, pp. 4075-4083. doi:10.1016/j.combustflame.2015.08.024

[19] Kierkegaard, A., Boij, S., and Efraimsson, G., "Simulations of the Scattering of Sound Waves at a Sudden Area Expansion," Journal of Sound and Vibration, Vol. 331, No. 5, 2012, pp. 1068-1083. doi:10.1016/j.jsv.2011.09.011

[20] Kierkegaard, A., Allam, S., Efraimsson, G., and Åbom, M., "Simulations of Whistling and the Whistling Potentiality of an In-Duct Orifice with Linear Aeroacoustics," Journal of Sound and Vibration, Vol. 331, No. 5, Feb. 2012, pp. 1084-1096. doi:10.1016/j.jsv.2011.10.028

[21] Gikadi, J., Ullrich, W. C., Sattelmayer, T., and Turrini, F., "Prediction of the Acoustic Losses of a Swirl Atomizer Nozzle Under Non-Reactive Conditions," ASME Turbo Expo 2013: Turbine Technical Conference and Exposition, American Soc. of Mechanical Engineers Paper V01BT04A035, New York, June 2013. doi:10.1115/gt2013-95449

[22] Stokes, G. G., "On the Effect of the Inertial Friction of Fluids on the Motions of Pendulums," Transactions of the Cambridge Philosophical Society, Vol. 9, 1851, pp. 8-23. doi: $10.1017 /$ cbo9780511702266.002

[23] Kirchhoff, G., "Ueber den Einfluss der Wärmeleitung in einem Gase auf die Schallbewegung," Annalen der Physik, Vol. 210, No. 6, 1868, pp. 177-193. doi:10.1002/(ISSN)1521-3889

[24] Nielsen, A. K., "Acoustic Resonators of Circular Cross-Section and with Axial Symmetry," Transactions of the Danish Academy of Technical Sciences, Vol. 10, 1949, pp. 9-70

[25] Searby, G., Nicole, A., Habiballah, M., and Laroche, E., "Prediction of the Efficiency of Acoustic Damping Cavities," Journal of Propulsion and Power, Vol. 24, No. 3, May 2008, pp. 516-523. doi: $10.2514 / 1.32325$

[26] Temkin, S., and Dobbins, R. A., "Attenuation and Dispersion of Sound by Particulate-Relaxation Processes," Journal of the Acoustical Society of America, Vol. 40, No. 2, 1966, p. 317. doi:10.1121/1.1910073

[27] Williams, F. A., Combustion Theory, Benjamin Cummings, Menlo Park, CA, 1985, pp. 304-315.

[28] Howe, M. S., "On the Theory of Unsteady High Reynolds Number Flow Through a Circular Aperture," Proceedings of the Royal Society of London, Series A: Mathematical, Physical and Engineering Sciences, Vol. 366, No. 1725, June 1979, pp. 205-223. doi:10.1098/rspa.1979.0048

[29] Jing, X., and Sun, X., "Effect of Plate Thickness on Impedance of Perforated Plates with Bias Flow," AIAA Journal, Vol. 38, No. 9, Jan. 2000, pp. 1573-1578. doi: $10.2514 / 2.1139$

[30] Mendez, S., and Eldredge, J., "Acoustic Modeling of Perforated Plates with Bias Flow for Large-Eddy Simulations," Journal of Computational Physics, Vol. 228, No. 13, July 2009, pp. 4757-4772. doi:10.1016/j.jcp.2009.03.026

[31] Tam, C. K. W., Ju, H., Jones, M. G., Watson, W. R., and Parrott, T. L., "A Computational and Experimental Study of Resonators in Three Dimensions," Journal of Sound and Vibration, Vol. 329, No. 24, Nov. 2010, pp. 5164-5193. doi:10.1016/j.jsv.2010.06.005

[32] Gullaud, E., and Nicoud, F., "Effect of Perforated Plates on the Acoustics of Annular Combustors," AIAA Journal, Vol. 50, No. 12, Dec. 2012, pp. 2629-2642. doi:10.2514/1.J050716

[33] Scarpato, A., Tran, N., Ducruix, S., and Schuller, T., "Modeling the Damping Properties of Perforated Screens Traversed by a Bias Flow and Backed by a Cavity at Low Strouhal Number,' Journal of Sound and 
Vibration, Vol. 331, No. 2, 2012, pp. 276-290. doi:10.1016/j.jsv.2011.09.005

[34] Fischer, A., Hirsch, C., and Sattelmayer, T., "Comparison of MultiMicrophone Transfer Matrix Measurements with Acoustic Network Models of Swirl Burners," Journal of Sound and Vibration, Vol. 298, Nos. 1-2, Nov. 2006, pp. 73-83. doi:10.1016/j.jsv.2006.04.040

[35] Föller, S., Polifke, W., and Tonon, D., "Aeroacoustic Characterization of T-Junctions Based on Large Eddy Simulation and System Identification," 16th AIAA/CEAS Aeroacoustics Conference, AIAA Paper 2010-3985, June 2010. doi:10.2514/6.2010-3985

[36] Karlsson, M., and Ábom, M., "Quasi-Steady Model of the Acoustic Scattering Properties of a T-Junction," Journal of Sound and Vibration, Vol. 330, No. 21, 2011, pp. 5131-5137. doi:10.1016/j.jsv.2011.05.012

[37] Howe, M., "The Dissipation of Sound at an Edge," Journal of Sound and Vibration, Vol. 70, No. 3, June 1980, pp. 407-411. doi:10.1016/0022-460X(80)90308-9

[38] Tam, C. K. W., Ju, H., Jones, M. G., Watson, W. R., and Parrott, T. L., "A Computational and Experimental Study of Slit Resonators," Journal of Sound and Vibration, Vol. 284, No. 3, June 2005, pp. $947-984$ doi:10.1016/j.jsv.2004.07.013

[39] Rienstra, S. W., and Hirschberg, A., An Introduction to Acoustics, Eindhoven Univ. of Technology, Eindhoven, 2003, pp. 30-32, 68-70.

[40] Åbom, M., "Measurement of the Scattering-Matrix of Acoustical Two-Ports," Mechanical Systems and Signal Processing, Vol. 5, No. 2, March 1991, pp. 89-104. doi:10.1016/0888-3270(91)90017-Y

[41] Paschereit, C. O., Polifke, W., Schuermans, B., and Mattson, O., "Measurement of Transfer Matrices and Source Terms of Premixed Flames," Journal of Engineering for Gas Turbines and Power, Vol. 124 No. 2, 2002, pp. 239-247. doi: $10.1115 / 1.1383255$

[42] Polifke, W., Poncet, A., Paschereit, C. O., and Doebbeling, K., "Reconstruction of Acoustic Transfer Matrices by Instationary Computational Fluid Dynamics," Journal of Sound and Vibration, Vol. 245, No. 3, Aug. 2001, pp. 483-510. doi:10.1006/jsvi.2001.3594

[43] Munjal, M. L., Acoustics of Ducts and Mufflers, Wiley, New York, 1986, pp. 75-85, 121-147.

[44] Gentemann, A., Fischer, A., Evesque, S., and Polifke, W., "Acoustic Transfer Matrix Reconstruction and Analysis for Ducts with Sudden Change of Area," 9th AIAA/CEAS Aeroacoustics Conference, AIAA Paper 2003-3142, May 2003. doi: $10.2514 / 6.2003-3142$

[45] Föller, S., and Polifke, W., "Identification of Aero-Acoustic Scattering Matrices from Large Eddy Simulation: Application to a Sudden Area Expansion of a Duct," Journal of Sound and Vibration, Vol. 331, No. 13, June 2012, pp. 3096-3113. doi:10.1016/j.jsv.2012.01.004

[46] Polifke, W., and Paschereit, C. O., "Determination of Thermo-Acoustic Transfer Matrices by Experiment and Computational Fluid Dynamics," ERCOFTAC Bulletin, 1998, p. 38

[47] Truffin, K., and Poinsot, T., "Comparison and Extension of Methods for Acoustic Identification of Burners," Combustion and Flame, Vol. 142, No. 4, Sept. 2005, pp. 388-400. doi:10.1016/j.combustflame.2005.04.001

[48] Bomberg, S., Emmert, T., and Polifke, W., "Thermal Versus Acoustic Response of Velocity Sensitive Premixed Flames," Proceedings of the Combustion Institute, Vol. 35, No. 3, 2015, pp. 3185-3192. doi:10.1016/j.proci.2014.07.032

[49] Strobio Chen, L., Witte, A., and Polifke, W., "Thermo-Acoustic Characterization of a Heat Exchanger in Cross Flow Using Compressible and Weakly Compressible Numerical Simulation," 22nd International Congress on Sound and Vibration (ICSV22), ICSV, Acoustical Soc. of Italy, July 2015, Paper 477, http://iiav.org/ icsv22.

[50] Jaensch, S., Emmert, T., Silva, C., and Polifke, W., "A Grey-Box Identification Approach for Thermoacoustic Network Models," ASME Turbo Expo 2014: Turbine Technical Conference and Exposition, American Soc. of Mechanical Engineers Paper V04BT04A051, New York, June 2014 doi:10.1115/gt2014-27034

[51] Bourgouin, J. F., Durox, D., Moeck, J. P., Schuller, T., and Candel, S., "Characterization and Modeling of a Spinning Thermoacoustic Instability in an Annular Combustor Equipped with Multiple Matrix Injectors," Journal of Engineering for Gas Turbines and Power,
Vol. 137, No. 2, June 2015, Paper 021503. doi:10.1115/gt2014-25067

[52] Campa, G., and Camporeale, S., "Eigenmode Analysis of the Thermoacoustic Combustion Instabilities Using a Hybrid Technique Based on the Finite Element Method and the Transfer Matrix Method," Advances in Applied Acoustics, Vol. 1, No. 1, 2012, pp. 1-14.

[53] Laera, D., Gentile, A., Camporeale, S. M., Bertolotto, E., Rofi, L., and Bonzani, F., "Numerical and Experimental Investigation of ThermoAcoustic Combustion Instability in a Longitudinal Combustion Chamber: Influence of the Geometry of the Plenum," ASME Turbo Expo 2015: Turbine Technical Conference and Exposition, American Soc. of Mechanical Engineers Paper V04AT04A028, June 2015. doi: $10.1115 / \mathrm{gt} 2015-42322$

[54] Nicoud, F., Benoit, L., Sensiau, C., and Poinsot, T., "Acoustic Modes in Combustors with Complex Impedances and Multidimensional Active Flames," AIAA Journal, Vol. 45, No. 2, Feb. 2007, pp. 426-441. doi: $10.2514 / 1.24933$

[55] Poinsot, T., Chatelier, T. L., Candel, S., and Esposito, E., "Experimental Determination of the Reflection Coefficient of a Premixed Flame in a Duct," Journal of Sound and Vibration, Vol. 107, No. 2, 1986, pp. $265-278$. doi:10.1016/0022-460X(86)90237-3

[56] Noiray, N., and Schuermans, B., "Deterministic Quantities Characterizing Noise Driven Hopf Bifurcations in Gas Turbine Combustors,' International Journal of Non-Linear Mechanics, Vol. 50, April 2013, pp. $152-163$. doi:10.1016/j.ijnonlinmec.2012.11.008

[57] Silva, C. F., Nicoud, F., Schuller, T., Durox, D., and Candel, S. "Combining a Helmholtz Solver with the Flame Describing Function to Assess Combustion Instability in a Premixed Swirled Combustor," Combustion and Flame, Vol. 160, No. 9, Sept. 2013, pp. 1743-1754. doi:10.1016/j.combustflame.2013.03.020

[58] Palies, P., Durox, D., Schuller, T., and Candel, S., "Nonlinear Combustion Instability Analysis Based on the Flame Describing Function Applied to Turbulent Premixed Swirling Flames," Combustion and Flame, Vol. 158, No. 10, 2011, pp. 1980-1991. doi:10.1016/j.combustflame.2011.02.012

[59] Holmberg, A., Ảbom, M., and Bodén, H., "Accurate Experimental Two-Port Analysis of Flow Generated Sound," Journal of Sound and Vibration, Vol. 330, No. 26, Dec. 2011, pp. 6336-6354. doi:10.1016/j.jsv.2011.07.041

[60] Schönfeld, T., "Steady and Unsteady Flow Simulations Using the Hybrid Flow Solver AVBP," AIAA Journal, Vol. 37, Jan. 1999, pp. 1378-1385. doi: $10.2514 / 3.14333$

[61] Selle, L., Lartigue, G., Poinsot, T., Koch, R., Schildmacher, K.-U., Krebs, W., Prade, B., Kaufmann, P., and Veynante, D., "Compressible Large Eddy Simulation of Turbulent Combustion in Complex Geometry on Unstructured Meshes," Combustion and Flame, Vol. 137, No. 4, 2004, pp. 489-505. doi:10.1016/j.combustflame.2004.03.008

[62] Mendez, S., and Nicoud, F., "Large-Eddy Simulation of a Bi-Periodic Turbulent Flow with Effusion,” Journal of Fluid Mechanics, Vol. 598, March 2008, pp. 27-65. doi:10.1017/S0022112007009664

[63] Duran, I., Leyko, M., Moreau, S., Nicoud, F., and Poinsot, T., "Computing Combustion Noise by Combining Large Eddy Simulations with Analytical Models for the Propagation of Waves Through Turbine Blades," Comptes Rendus de l'Académie des Sciences, Series IIB: Mécanique, Vol. 341, Nos. 1-2, Jan. 2013, pp. 131-140. doi:10.1016/j.crme.2012.10.012

[64] Motheau, E., Nicoud, F., and Poinsot, T., "Mixed Acoustic-Entropy Combustion Instabilities in Gas Turbines," Journal of Fluid Mechanics, Vol. 749, June 2014, pp. 542-576. doi:10.1017/jfm.2014.245

[65] Colin, O., and Rudgyard, M., "Development of High-Order TaylorGalerkin Schemes for Unsteady Calculations," Journal of Computational Physics, Vol. 162, No. 2, 2000, pp. 338-371. doi:10.1006/jcph.2000.6538

[66] Nicoud, F., Baya Toda, H., Cabrit, O., Bose, S., and Lee, J., "Using Singular Values to Build a Subgrid-Scale Model for Large Eddy Simulations," Physics of Fluids, Vol. 23, No. 8, 2011 , Paper 085106. doi:10.1063/1.3623274

[67] Baya Toda, H., Cabrit, O., Truffin, K., Bruneaux, G., and Nicoud, F. "Assessment of Subgrid-Scale Models with a Large-Eddy SimulationDedicated Experimental Database: The Pulsatile Impinging Jet in Turbulent Cross-Flow," Physics of Fluids, Vol. 26, No. 7, July 2014, Paper 075108 .

doi: $10.1063 / 1.4890855$ 
[68] Poinsot, T., and Lele, S., "Boundary Conditions for Direct Simulations of Compressible Viscous Flows," Journal of Computational Physics, Vol. 101, No. 1, 1992, pp. 104-129. doi:10.1016/0021-9991(92)90046-2

[69] Paschereit, C. O., and Polifke, W.. "Characterization of Lean Premixed Gas-Turbine Burners as Acoustic Multi-Ports," Bulletin of the American Physical Society/Division of Fluid Dynamics, Annual Meeting, San Francisco, CA, 1997.

[70] Bauerheim, M., "Theoretical and Numerical Study of Symmetry Breaking Effects on Azimuthal Thermoacoustic Modes in Annular Combustors," Ph.D. Thesis, Institut National Polytechnique de Toulouse, Toulouse, 2014

[71] Levine, H., and Schwinger, J., "On the Radiation of Sound from an Unflanged Circular Pipe," Physical Review, Vol. 73, No. 4, Feb. 1948, pp. 383-406.

doi:10.1103/PhysRev.73.383

[72] Schmid, P. J., "Dynamic Mode Decomposition of Numerical and Experimental Data," Journal of Fluid Mechanics, Vol. 656, July 2010, pp. $5-28$.

doi:10.1017/S0022112010001217

[73] Aurégan, Y., and Starobinski, R., "Determination of Acoustical Energy Dissipation/Production Potentiality from the Acoustical Transfer Functions of a Multiport," Acta Acustica United with Acustica, Vol. 85, No. 6, 1999, pp. 788-792. 